\title{
Study of $b$-quark mass effects in multijet topologies with the DELPHI detector at LEP
}

\section{The DELPHI Collaboration}

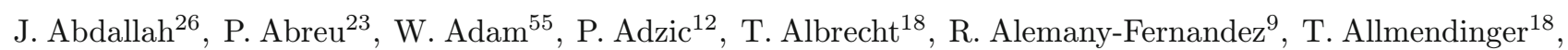
P.P. Allport ${ }^{24}$, U. Amaldi ${ }^{30}$, N. Amapane ${ }^{48}$, S. Amato ${ }^{52}$, E. Anashkin ${ }^{37}$, A. Andreazza ${ }^{29}$, S. Andringa ${ }^{23}$, N. Anjos ${ }^{23}$, P. Antilogus ${ }^{26}$, W-D. Apel ${ }^{18}$, Y. Arnoud ${ }^{15}$, S. Ask ${ }^{9}$, B. Asman ${ }^{47}$, J.E. Augustin ${ }^{26}$, A. Augustinus ${ }^{9}$, P. Baillon ${ }^{9}$, A. Ballestrero ${ }^{49}$, P. Bambade ${ }^{21}$, R. Barbier ${ }^{28}$, D. Bardin ${ }^{17}$, G.J. Barker ${ }^{57}$, A. Baroncelli ${ }^{40}$, M. Battaglia ${ }^{9}$, M. Baubillier ${ }^{26}$, K-H. Becks ${ }^{58}$, M. Begalli ${ }^{7}$, A. Behrmann ${ }^{58}$, E. Ben-Haim ${ }^{21}$, N. Benekos ${ }^{33}$, A. Benvenuti ${ }^{5}$, C. Berat ${ }^{15}$, M. Berggren ${ }^{26}$, D. Bertrand ${ }^{2}$, M. Besancon ${ }^{41}$, N. Besson ${ }^{41}$, D. Bloch ${ }^{10}$, M. Blom ${ }^{32}$, M. Bluj ${ }^{56}$, M. Bonesini ${ }^{30}$, M. Boonekamp ${ }^{41}$, P.S.L. Booth ${ }^{24, a}$, G. Borisov ${ }^{22}$, O. Botner ${ }^{53}$, B. Bouquet ${ }^{21}$, T.J.V. Bowcock ${ }^{24}$, I. Boyko ${ }^{17}$, M. Bracko ${ }^{44}$, R. Brenner ${ }^{53}$, E. Brodet ${ }^{36}$, P. Bruckman ${ }^{19}$, J.M. Brunet ${ }^{8}$, B. Buschbeck ${ }^{55}$, P. Buschmann ${ }^{58}$, M. Calvi ${ }^{30}$, T. Camporesi ${ }^{9}$, V. Canale ${ }^{39}$, F. Carena ${ }^{9}$, N. Castro ${ }^{23}$, F. Cavallo ${ }^{5}$, M. Chapkin ${ }^{43}$, Ph. Charpentier ${ }^{9}$, P. Checchia ${ }^{37}$, R. Chierici ${ }^{9}$, P. Chliapnikov ${ }^{43}$, J. Chudoba ${ }^{9}$, S.U. Chung ${ }^{9}$, K. Cieslik ${ }^{19}$, P. Collins ${ }^{9}$, R. Contri ${ }^{14}$, G. Cosme ${ }^{21}$, F. Cossutti ${ }^{50}$, M.J. Costa ${ }^{54}$, D. Crennell ${ }^{38}$, J. Cuevas ${ }^{35}$, J. D'Hondt ${ }^{2}$, T. da Silva ${ }^{52}$, W. Da Silva ${ }^{26}$, G. Della Ricca ${ }^{50}$, A. De Angelis ${ }^{51}$, W. De Boer ${ }^{18}$, C. De Clercq ${ }^{2}$, B. De Lotto ${ }^{51}$, N. De Maria ${ }^{48}$, A. De Min ${ }^{37}$, L. de Paula ${ }^{52}$, L. Di Ciaccio ${ }^{39}$, A. Di Simone ${ }^{40}$, K. Doroba ${ }^{56}$, J. Drees ${ }^{58,9}$, G. Eigen ${ }^{4}$, T. Ekelof ${ }^{53}$, M. Ellert ${ }^{53}$, M. Elsing ${ }^{9}$, M.C. Espirito Santo ${ }^{23}$, G. Fanourakis ${ }^{12}$, D. Fassouliotis ${ }^{12,3}$, M. Feindt ${ }^{18}$, J. Fernandez ${ }^{42}$, A. Ferrer ${ }^{54}$, F. Ferro ${ }^{14}$, U. Flagmeyer ${ }^{58}$, H. Foeth ${ }^{9}$, E. Fokitis ${ }^{33}$, F. Fulda-Quenzer ${ }^{21}$, J. Fuster ${ }^{54}$, M. Gandelman ${ }^{52}$, C. Garcia ${ }^{54}$, Ph. Gavillet ${ }^{9}$, E. Gazis ${ }^{33}$, R. Gokieli ${ }^{9}, 56$, B. Golob ${ }^{44,46}$, G. Gomez-Ceballos ${ }^{42}$, P. Goncalves ${ }^{23}$, E. Graziani ${ }^{40}$, G. Grosdidier ${ }^{21}$, K. Grzelak ${ }^{56}$, J. Guy ${ }^{38}$, C. Haag ${ }^{18}$, A. Hallgren ${ }^{53}$, K. Hamacher ${ }^{58}$, K. Hamilton ${ }^{36}$, S. Haug ${ }^{34}$, F. Hauler ${ }^{18}$, V. Hedberg ${ }^{27}$, M. Hennecke ${ }^{18}$, H. Herr ${ }^{9}$, a , J. Hoffman ${ }^{56}$, S-O. Holmgren ${ }^{47}$, P.J. Holt ${ }^{9}$, M.A. Houlden ${ }^{24}$, J.N. Jackson ${ }^{24}$, G. Jarlskog ${ }^{27}$, P. Jarry ${ }^{41}$, D. Jeans ${ }^{36}$, E.K. Johansson ${ }^{47}$, P. Jonsson ${ }^{28}$, C. Joram ${ }^{9}$, L. Jungermann ${ }^{18}$, F. Kapusta ${ }^{26}$, S. Katsanevas ${ }^{28}$, E. Katsoufis ${ }^{33}$, G. Kernel ${ }^{44}$, B.P. Kersevan ${ }^{44,46}$, U. Kerzel ${ }^{18}$, B.T. King ${ }^{24}$, N.J. Kjaer ${ }^{9}$, P. Kluit ${ }^{32}$, P. Kokkinias ${ }^{12}$, C. Kourkoumelis ${ }^{3}$, O. Kouznetsov ${ }^{17}$, Z. Krumstein ${ }^{17}$, M. Kucharczyk ${ }^{19}$, J. Lamsa ${ }^{1}$, G. Leder ${ }^{55}$, F. Ledroit ${ }^{15}$, L. Leinonen ${ }^{47}$, R. Leitner ${ }^{31}$, J. Lemonne ${ }^{2}$, V. Lepeltier ${ }^{21, a}$, T. Lesiak ${ }^{19}$, W. Liebig ${ }^{58}$, D. Liko ${ }^{55}$, A. Lipniacka ${ }^{47}$, J.H. Lopes ${ }^{52}$, J.M. Lopez ${ }^{35}$, D. Loukas ${ }^{12}$, P. Lutz ${ }^{41}$, L. Lyons ${ }^{36}$, J. MacNaughton ${ }^{55}$, A. Malek ${ }^{58}$, S. Maltezos ${ }^{33}$, F. Mandl ${ }^{55}$, J. Marco ${ }^{42}$, R. Marco ${ }^{42}$, B. Marechal ${ }^{52}$, M. Margoni ${ }^{37}$, J-C. Marin ${ }^{9}$, C. Mariotti ${ }^{9}$, A. Markou ${ }^{12}$, C. Martinez-Rivero ${ }^{42}$, J. Masik ${ }^{13}$, N. Mastroyiannopoulos ${ }^{12}$, F. Matorras ${ }^{42}$, C. Matteuzzi ${ }^{30}$, F. Mazzucato ${ }^{37}$, M. Mazzucato ${ }^{37}$, R. Mc Nulty ${ }^{24}$, C. Meroni ${ }^{29}$, E. Migliore ${ }^{48}$, W. Mitaroff ${ }^{55}$, U. Mjoernmark ${ }^{27}$, T. Moa ${ }^{47}$, M. Moch ${ }^{18}$, K. Moenig ${ }^{9,11}$, R. Monge ${ }^{14}$, J. Montenegro ${ }^{32}$, D. Moraes ${ }^{52}$, S. Moreno ${ }^{23}$, P. Morettini ${ }^{14}$, U. Mueller ${ }^{58}$, K. Muenich ${ }^{58}$, M. Mulders ${ }^{32}$, L. Mundim', W. Murray ${ }^{38}$, B. Muryn ${ }^{20}$, G. Myatt ${ }^{36}$, T. Myklebust ${ }^{34}$, M. Nassiakou ${ }^{12}$, F. Navarria ${ }^{5}$, K. Nawrocki ${ }^{56}$, R. Nicolaidou ${ }^{41}$, M. Nikolenko $^{17,10}$,

A. Oblakowska-Mucha ${ }^{20}$, V. Obraztsov ${ }^{43}$, A. Olshevski ${ }^{17}$, A. Onofre ${ }^{23}$, R. Orava ${ }^{16}$, K. Osterberg ${ }^{16}$, A. Ouraou ${ }^{41}$, A. Oyanguren ${ }^{54}$, M. Paganoni ${ }^{30}$, S. Paiano ${ }^{5}$, J.P. Palacios ${ }^{24}$, H. Palka ${ }^{19}$, Th.D. Papadopoulou ${ }^{33}$, L. Pape ${ }^{9}$, C. Parkes $^{25}$, F. Parodi ${ }^{14}$, U. Parzefall ${ }^{9}$, A. Passeri ${ }^{40}$, O. Passon ${ }^{58}$, L. Peralta ${ }^{23}$, V. Perepelitsa ${ }^{54}$, A. Perrotta ${ }^{5}$, A. Petrolini ${ }^{14}$, J. Piedra ${ }^{42}$, L. Pieri ${ }^{40}$, F. Pierre ${ }^{41}$, M. Pimenta ${ }^{23}$, E. Piotto ${ }^{9}$, T. Podobnik ${ }^{44,46}$, V. Poireau ${ }^{9}$, M.E. Pol ${ }^{6}$, G. Polok ${ }^{19}$, V. Pozdniakov ${ }^{17}$, N. Pukhaeva ${ }^{17}$, A. Pullia ${ }^{30}$, J. Rames ${ }^{13}$, A. Read ${ }^{34}$, P. Rebecchi ${ }^{9}$, J. Rehn ${ }^{18}$, D. Reid ${ }^{32}$, R. Reinhardt ${ }^{58}$, P. Renton ${ }^{36}$, F. Richard ${ }^{21}$, J. Ridky ${ }^{13}$, M. Rivero ${ }^{42}$, D. Rodriguez ${ }^{42}$, A. Romero ${ }^{48}$, P. Ronchese ${ }^{37}$, P. Roudeau ${ }^{21}$, T. Rovelli ${ }^{5}$, V. Ruhlmann-Kleider ${ }^{41}$, D. Ryabtchikov ${ }^{43}$, A. Sadovsky ${ }^{17}$, L. Salmi ${ }^{16}$, J. Salt ${ }^{54}$, C. Sander ${ }^{18}$, A. Savoy-Navarro ${ }^{26}$, U. Schwickerath ${ }^{9}$, R. Sekulin ${ }^{38}$, M. Siebel ${ }^{58}$, A. Sisakian ${ }^{17}$, G. Smadja ${ }^{28}$, O. Smirnova ${ }^{27}$, A. Sokolov ${ }^{43}$, A. Sopczak ${ }^{22}$, R. Sosnowski ${ }^{56}$, T. Spassov ${ }^{9}$, M. Stanitzki ${ }^{18}$, A. Stocchi ${ }^{21}$, J. Strauss ${ }^{55}$, B. Stugu ${ }^{4}$, M. Szczekowski ${ }^{56}$, M. Szeptycka ${ }^{56}$, T. Szumlak ${ }^{20}$, T. Tabarelli ${ }^{30}$, F. Tegenfeldt $^{53}$, J. Timmermans ${ }^{32, b}$, L. Tkatchev ${ }^{17}$, M. Tobin ${ }^{24}$, S. Todorovova ${ }^{13}$, B. Tome ${ }^{23}$, A. Tonazzo ${ }^{30}$, P. Tortosa ${ }^{54}$, P. Travnicek ${ }^{13}$, D. Treille ${ }^{9}$, G. Tristram ${ }^{8}$, M. Trochimczuk ${ }^{56}$, C. Troncon $^{29}$, M-L. Turluer ${ }^{41}$, I.A. Tyapkin ${ }^{17}$, P. Tyapkin ${ }^{17}$, S. Tzamarias ${ }^{12}$, V. Uvarov ${ }^{43}$, G. Valenti ${ }^{5}$, P. Van Dam ${ }^{32}$, J. Van Eldik ${ }^{9}$, N. van Remortel ${ }^{16}$, I. Van Vulpen ${ }^{9}$, G. Vegni ${ }^{29}$, F. Veloso ${ }^{23}$, W. Venus ${ }^{38}$, P. $\operatorname{Verdier}^{28}$, V. Verzi ${ }^{39}$, D. Vilanova ${ }^{41}$, L. Vitale $^{50}$, V. Vrba ${ }^{13}$, H. Wahlen ${ }^{58}$, A.J. Washbrook ${ }^{24}$, C. Weiser ${ }^{18}$, D. Wicke ${ }^{9}$, J. Wickens ${ }^{2}$, G. Wilkinson ${ }^{36}$, M. Winter ${ }^{10}$, M. Witek ${ }^{19}$, O. Yushchenko ${ }^{43}$, A. Zalewska ${ }^{19}$, P. Zalewski ${ }^{56}$, D. Zavrtanik ${ }^{45}$, V. Zhuravlov ${ }^{17}$, N.I. Zimin ${ }^{17}$, A. Zintchenko ${ }^{17}$, M. Zupan ${ }^{12}$

${ }^{1}$ Department of Physics and Astronomy, Iowa State University, Ames IA 50011-3160, USA

${ }^{2}$ IIHE, ULB-VUB, Pleinlaan 2, 1050 Brussels, Belgium 
3 Physics Laboratory, University of Athens, Solonos Str. 104, 10680 Athens, Greece

4 Department of Physics, University of Bergen, Allégaten 55, 5007 Bergen, Norway

5 Dipartimento di Fisica, Università di Bologna and INFN, Via Irnerio 46, 40126 Bologna, Italy

${ }^{6}$ Centro Brasileiro de Pesquisas Físicas, rua Xavier Sigaud 150, 22290 Rio de Janeiro, Brazil

7 Inst. de Física, Univ. Estadual do Rio de Janeiro, rua São Francisco Xavier 524, Rio de Janeiro, Brazil

8 Collège de France, Lab. de Physique Corpusculaire, IN2P3-CNRS, 75231 Paris Cedex 05, France

${ }^{9}$ CERN, 1211 Geneva 23, Switzerland

${ }^{10}$ Institut de Recherches Subatomiques, IN2P3 - CNRS/ULP - BP20, 67037 Strasbourg Cedex, France

11 DESY-Zeuthen, Platanenallee 6, 15735 Zeuthen, Germany

12 Institute of Nuclear Physics, N.C.S.R. Demokritos, P.O. Box 60228, 15310 Athens, Greece

13 FZU, Inst. of Phys. of the C.A.S. High Energy Physics Division, Na Slovance 2, 182 21, Praha 8, Czech Republic

14 Dipartimento di Fisica, Università di Genova and INFN, Via Dodecaneso 33, 16146 Genova, Italy

15 Institut des Sciences Nucléaires, IN2P3-CNRS, Université de Grenoble 1, 38026 Grenoble Cedex, France

${ }^{16}$ Helsinki Institute of Physics and Department of Physical Sciences, P.O. Box 64, 00014 University of Helsinki, Finland

17 Joint Institute for Nuclear Research, Dubna, Head Post Office, P.O. Box 79, 101000 Moscow, Russian Federation

18 Institut für Experimentelle Kernphysik, Universität Karlsruhe, Postfach 6980, 76128 Karlsruhe, Germany

19 Institute of Nuclear Physics PAN, Ul. Radzikowskiego 152, 31142 Krakow, Poland

${ }^{20}$ Faculty of Physics and Nuclear Techniques, University of Mining and Metallurgy, 30055 Krakow, Poland

${ }^{21}$ LAL, Univ Paris-Sud, CNRS/IN2P3, Orsay, France

22 School of Physics and Chemistry, University of Lancaster, Lancaster LA1 4YB, UK

${ }^{23}$ LIP, IST, FCUL - Av. Elias Garcia, 14-1 ${ }^{\circ}, 1000$ Lisboa Codex, Portugal

24 Department of Physics, University of Liverpool, P.O. Box 147, Liverpool L69 3BX, UK

25 Dept. of Physics and Astronomy, Kelvin Building, University of Glasgow, Glasgow G12 8QQ, UK

26 LPNHE, IN2P3-CNRS, Univ. Paris VI et VII, Tour 33 (RdC), 4 place Jussieu, 75252 Paris Cedex 05, France

27 Department of Physics, University of Lund, Sölvegatan 14, 22363 Lund, Sweden

28 Université Claude Bernard de Lyon, IPNL, IN2P3-CNRS, 69622 Villeurbanne Cedex, France

29 Dipartimento di Fisica, Università di Milano and INFN-MILANO, Via Celoria 16, 20133 Milan, Italy

30 Dipartimento di Fisica, Univ. di Milano-Bicocca and INFN-MILANO, Piazza della Scienza 3, 20126 Milan, Italy

31 IPNP of MFF, Charles Univ., Areal MFF, V Holesovickach 2, 18000, Praha 8, Czech Republic

32 NIKHEF, Postbus 41882, 1009 DB Amsterdam, The Netherlands

33 National Technical University, Physics Department, Zografou Campus, 15773 Athens, Greece

34 Physics Department, University of Oslo, Blindern, 0316 Oslo, Norway

35 Dpto. Fisica, Univ. Oviedo, Avda. Calvo Sotelo s/n, 33007 Oviedo, Spain

36 Department of Physics, University of Oxford, Keble Road, Oxford OX1 3RH, UK

37 Dipartimento di Fisica, Università di Padova and INFN, Via Marzolo 8, 35131 Padua, Italy

38 Rutherford Appleton Laboratory, Chilton, Didcot OX11 OQX, UK

39 Dipartimento di Fisica, Università di Roma II and INFN, Tor Vergata, 00173 Rome, Italy

40 Dipartimento di Fisica, Università di Roma III and INFN, Via della Vasca Navale 84, 00146 Rome, Italy

41 DAPNIA/Service de Physique des Particules, CEA-Saclay, 91191 Gif-sur-Yvette Cedex, France

42 Instituto de Fisica de Cantabria (CSIC-UC), Avda. los Castros s/n, 39006 Santander, Spain

43 Inst. for High Energy Physics, Serpukov P.O. Box 35, Protvino, (Moscow Region), Russian Federation

44 J. Stefan Institute, Jamova 39, 1000 Ljubljana, Slovenia

45 Laboratory for Astroparticle Physics, University of Nova Gorica, Kostanjeviska 16a, 5000 Nova Gorica, Slovenia

46 Department of Physics, University of Ljubljana, 1000 Ljubljana, Slovenia

47 Fysikum, Stockholm University, Box 6730, 11385 Stockholm, Sweden

48 Dipartimento di Fisica Sperimentale, Università di Torino and INFN, Via P. Giuria 1, 10125 Turin, Italy

49 INFN, Sezione di Torino and Dipartimento di Fisica Teorica, Università di Torino, Via Giuria 1, 10125 Turin, Italy

50 Dipartimento di Fisica, Università di Trieste and INFN, Via A. Valerio 2, 34127 Trieste, Italy

51 Istituto di Fisica, Università di Udine and INFN, 33100 Udine, Italy

52 Univ. Federal do Rio de Janeiro, C.P. 68528 Cidade Univ., Ilha do Fundão 21945-970 Rio de Janeiro, Brazil

53 Department of Radiation Sciences, University of Uppsala, P.O. Box 535, 75121 Uppsala, Sweden

54 IFIC, Valencia-CSIC, and D.F.A.M.N., U. de Valencia, Avda. Dr. Moliner 50, 46100 Burjassot (Valencia), Spain

55 Institut für Hochenergiephysik, Österr. Akad. d. Wissensch., Nikolsdorfergasse 18, 1050 Vienna, Austria

${ }^{56}$ Inst. Nuclear Studies and University of Warsaw, Ul. Hoza 69, 00681 Warsaw, Poland

57 Now at University of Warwick, Coventry CV4 7AL, UK

58 Fachbereich Physik, University of Wuppertal, Postfach 100127, 42097 Wuppertal, Germany

Received: 6 June 2007 / Revised version: 22 April 2008 /

Published online: 28 May 2008 - (C) Springer-Verlag / Società Italiana di Fisica 2008 


\begin{abstract}
The effect of the heavy $b$-quark mass on the two, three and four-jet rates is studied using LEP data collected by the DELPHI experiment at the $Z$ peak in 1994 and 1995 . The rates of $b$-quark jets and light quark jets $(\ell=u d s)$ in events with $n=2,3$, and 4 jets, together with the ratio of two and four-jet rates of $b$-quarks with respect to light-quarks, $R_{n}^{b \ell}$, have been measured with a double-tag technique using the CAMBRIDGE jetclustering algorithm. A comparison between experimental results and theory (matrix element or Monte Carlo event generators such as PYTHIA, HERWIG and ARIADNE) is done after the hadronisation phase.

Using the four-jet observable $R_{4}^{b \ell}$, a measurement of the $b$-quark mass using massive leading-order calculations gives:
\end{abstract}

$$
m_{b}\left(M_{Z}\right)=3.76 \pm 0.32(\text { stat }) \pm 0.17(\text { syst }) \pm 0.22(\text { had }) \pm 0.90(\text { theo }) \mathrm{GeV} / c^{2}
$$

This result is compatible with previous three-jet determinations at the $M_{Z}$ energy scale and with low energy mass measurements evolved to the $M_{Z}$ scale using QCD renormalisation group equations.

\section{Introduction}

Mass corrections to the $Z \rightarrow b \bar{b}$ coupling are of order $\left(m_{b}^{2} / M_{Z}^{2}\right)$, which is too small to be measured at LEP and SLC. For some inclusive observables, like jet-rates, the effect is enhanced as $\left(m_{b}^{2} / M_{Z}^{2}\right) / y_{\text {cut }}$, where $y_{\text {cut }}$ is the jet resolution parameter [1]. The effect of the $b$-quark mass in the production of three-jet event topologies at the $Z$ peak has for instance already been measured at LEP and SLC [2-6]. Multi-jet topologies with $b$-quarks appear both as signal and background in searches and precision measurements at current and future colliders. Their study, together with that of the gluon emission from massive quarks, is an effective tool to probe the fundamental short-distance QCD features of the Standard Model and is important to test the modelling of $b$ and light-quark jets available in calculations and generators.

This study generalizes the methods described in $[2,3,7]$ and presents the measurement of the normalized $n$-jet production partial widths for $Z$-decays into $b$-quark or light quark pairs:

$$
R_{n=2,3,4}^{q}\left(y_{\text {cut }}\right)=\left[\frac{\Gamma_{n}\left(y_{\text {cut }}\right)}{\Gamma_{\text {tot }}}\right]^{Z \rightarrow q \bar{q}} \quad q=b, \ell(\ell=u d s),
$$

depending on the $y_{\text {cut }}$ value of the CAMBRIDGE jetclustering algorithm [8] which is used here.

The effect of the heavy $b$-quark mass on jet rates is studied by measuring the double-ratio observable:

$$
R_{n=2,3,4}^{b \ell}=R_{n}^{b} / R_{n}^{\ell} .
$$

The DELPHI data collected during the years 1994 and 1995 at a centre-of-mass energy of $\sqrt{s} \approx M_{Z}$ have been analysed. Experimental results are compared to the hadronic final state simulated by the fragmentation models of PYTHIA 6.156 [9, 10], HERWIG $6.2[11,12]$ and ARIADNE 4.08 [13] and to matrix element (ME) calculations folded with a hadronisation correction. Therefore, the data are corrected for detector and kinematical effects, while ME calculations, computed at parton level, are corrected for hadronisation.

\footnotetext{
${ }^{a}$ deceased

b e-mail: jan.timmermans@cern.ch
}

In order to extract the $b$-quark mass information from $R_{n}^{b \ell}$ measurements, massive ME calculations performed in terms of both the pole mass $M_{b}$ and the running mass $m_{b}(\mu)$ are used. Jet-rate calculations are only available to $\mathcal{O}\left(\alpha_{\mathrm{s}}^{2}\right)$ [14-16], therefore massive four-jet observables can only be described to leading-order (LO) accuracy. The $b$-quark mass obtained from $R_{4}^{b \ell}$ using such LO calculations is compared to the three-jet results [7] and to mass values at threshold [17] evolved to the $M_{Z}$ scale using renormalisation group equations (RGE). An approximate massless NLO correction is also tried as an improvement.

The precision of $b$-mass measurements from three-jet events is limited by systematic uncertainties (hadronisation, $b$-tagging and theory). The four-jet observable $R_{4}^{b \ell}$ has a larger statistical error but its sensitivity to the $b$-quark mass is higher because, most probably, the emission of two gluons is involved. The four-jet topology thus provides a complementary measurement in which the systematic uncertainties can be expected to be partly different. In this analysis, flavour jet-rates are measured using a double-tag technique which measures signal and background efficiencies from data in a self-calibrating way, reducing the systematics and allowing for a useful crosscheck of previous measurements [7].

\section{The DELPHI detector}

DELPHI was a hermetic detector located at the LEP accelerator, with a superconducting solenoid providing a uniform magnetic field of $1.23 \mathrm{~T}$ parallel to the beam axis throughout the central tracking device volume. A detailed description of its design and performance is presented in $[22,23]$.

In the DELPHI coordinate system, the $z$-axis is oriented along the direction of the electron beam. The polar angle $\theta$ is measured with respect to the $z$-axis, $\phi$ is the azimuthal angle in the plane transverse to the $z$-axis and $R=\sqrt{x^{2}+y^{2}}$ is the radial coordinate.

The main tracking devices in DELPHI were the silicon Vertex Detector (VD), a jet chamber Inner Detector (ID) and a time projection chamber (TPC). They were located in the immediate vicinity of the interaction region to reduce the amount of material between the beam and the detector. At a larger distance, the tracking was completed 
by a drift chamber Outer Detector (OD) covering the barrel region $\left(40^{\circ} \leq \theta \leq 140^{\circ}\right)$ and two sets of drift chambers, FCA and FCB, located in the endcaps.

The VD was the detector closest to the interaction point. In 1994 and 1995 it consisted of three coaxial cylinders, the inner and outermost ones consisting of doublesided detectors with orthogonal strips, allowing the measurement of both $R \phi$ and $z$ coordinates.

Electron and photon identification was provided by electromagnetic calorimeters: the High Density Projection Chamber (HPC) in the barrel and a lead-glass calorimeter (FEMC) in the endcaps. Hadronic energy was measured in the hadronic calorimeter (HCAL).

\section{Data analysis}

First, the sample of $Z$ hadronic decays, i.e. $Z \rightarrow q \bar{q}$ events was selected. Then the different jet-topologies were identified using the CAMBRIDGE jet-clustering algorithm $[8]^{1}$, and $b$ and light-quark samples were separated using the DELPHI flavour tagging methods, based on properties of the long-lived heavy $B$-hadrons. Experimental results were then corrected for detector and acceptance effects in two different ways, depending on the observable and topology, as explained in Sect. 3.3. Matrix element and event generator predictions were corrected for hadronisation effects from the parton to the hadron level. The parton level is defined as the final state of the parton shower (in PYTHIA and HERWIG) or dipole cascade (in ARIADNE) in the simulation, before hadronisation. These corrections are discussed in Sect. 4.

\subsection{Event selection}

Total numbers of 1484000 and 750000 hadronic $Z$ boson decays, collected at the $Z$ resonance by DELPHI during the years 1994 and 1995, respectively, have been analysed in order to study mass effects in multi-jet topologies. ${ }^{2}$

Hadronic events were selected in the same way as in $[2,3]$ (see Table 1 , upper):

- Charged and neutral particles were reconstructed as tracks and energy depositions in the detector. A first selection was applied to ensure a reliable determination of their momenta and energies.

- The information from the accepted tracks was combined event-by-event and hadronic events were selected according to global event properties.

Finally, a total sample of $1150000 Z$ hadronic decays was selected. Then jets were reconstructed with the CAMBRIDGE algorithm. In order to reduce the impact of particle losses and wrong energy-momentum assignment

\footnotetext{
1 In our analysis, the values of the ordering and resolution parameters were taken to be equal, $v_{i j}=y_{i j}$.

2 Earlier data samples were not considered as the VD setup was less complete and resulted in a less precise flavour identification, which is crucial for this analysis.
}

Table 1. (Upper) Particle and event selections: $p_{\text {ch }}$ is the momentum of charged particles, $L$ their measured track length, $d$ their impact parameter with respect to the interaction point and $q_{i}$ their charge, $E_{\mathrm{cl}}$ is the energy of neutral clusters in the calorimeters, $N_{\mathrm{ch}}$ is the number of charged particles and $E_{\mathrm{ch}}$ their total energy in the event. (Lower) Kinematical selections for jets in accepted events: $\theta_{\text {thrust }}$ is the polar angle of the thrust of the event, $N_{j}^{\text {ch }}$ the charged multiplicity in the jet, $E_{j}$ the jet energy and $\theta_{j}$ the angle between the jet and the beam axis. For three-jet events, an additional planarity cut is applied on the sum of all jet pair angles, $\phi_{i j}$

\begin{tabular}{|c|c|}
\hline $\begin{array}{l}\text { Charged } \\
\text { Particle } \\
\text { Selection }\end{array}$ & $\begin{array}{c}p_{\mathrm{ch}} \geq 0.1 \mathrm{GeV} / c \\
25^{\circ} \leq \theta \leq 155^{\circ} \\
L \geq 50 \mathrm{~cm} \\
d \leq 5 \mathrm{~cm} \text { in } R \phi \text { plane } \\
d \leq 10 \mathrm{~cm} \text { in } z \text { direction }\end{array}$ \\
\hline Neutral & $E_{\mathrm{cl}}^{\mathrm{HPC}} \geq 0.5 \mathrm{GeV}, 40^{\circ} \leq \theta \leq 140^{\circ}$ \\
\hline Cluster & $E_{\mathrm{cl}}^{\mathrm{FEMC}} \geq 0.5 \mathrm{GeV}, 8^{\circ} \leq \theta \leq 36^{\circ}$ \\
\hline Selection & $\begin{array}{c}E_{\mathrm{cl}}^{\mathrm{FEMC}} \geq 0.5 \mathrm{GeV}, 144^{\circ} \leq \theta \leq 172^{\circ} \\
E_{\mathrm{cl}}^{\mathrm{HAC}} \geq 1 \mathrm{GeV}, 10^{\circ} \leq \theta \leq 170^{\circ}\end{array}$ \\
\hline $\begin{array}{l}\text { Event } \\
\text { Selection }\end{array}$ & $\begin{array}{c}N_{\mathrm{ch}} \geq 5 \\
E_{\mathrm{ch}} \geq 15 \mathrm{GeV} \\
\left|\sum_{i} q_{i}\right| \leq 6, i=1, \ldots, N_{\mathrm{ch}} \\
\text { No particle with } p_{\mathrm{ch}} \geq 40 \mathrm{GeV} / c\end{array}$ \\
\hline 2 -jet & $45^{\circ} \leq \theta_{\text {thrust }} \leq 135^{\circ}$ \\
\hline 3 -jet & $\begin{array}{c}45^{\circ} \leq \theta_{\text {thrust }} \leq 135^{\circ} \\
N_{j}^{\text {ch }} \geq 1 \\
E_{j} \geq 1 \mathrm{GeV} \\
25^{\circ} \leq \theta_{j} \leq 155^{\circ} \\
\sum_{i j} \phi_{i j} \geq 359^{\circ}, i<j\end{array}$ \\
\hline 4 -jet & $\begin{array}{c}32^{\circ} \leq \theta_{\text {thrust }} \leq 148^{\circ} \\
N_{j}^{\text {ch }} \geq 1 \\
E_{j} \geq 1 \mathrm{GeV} \\
25^{\circ} \leq \theta_{j} \leq 155^{\circ}\end{array}$ \\
\hline
\end{tabular}

to jets, further kinematical selections were applied, which were slightly different for each jet topology (see Table 1 , lower).

Simulated events were produced with the DELPHI simulation program DELSIM [23], based on PYTHIA 7.3 tuned to DELPHI data $[24,25]$, and were then passed through the same reconstruction and analysis chain as the experimental data. The simulated events were reweighted in order to reproduce the measured rates of $b \bar{b}$ and $c \bar{c}$ quark pairs arising from the gluon splitting processes [26] $\left(g_{b \bar{b}}=0.00254 \pm 0.00051, g_{c \bar{c}}=0.0296 \pm 0.0038\right)$, which are significantly larger than those in the standard simulation.

\section{$3.2 b$-tagging}

The identification of $b$-quark events in DELPHI was based on the properties of a $B$-hadron such as its large mass and the large impact parameter of its decay products. A jet estimator variable $X_{\text {jet }}$ was built as an optimal combination of five discriminating variables [27]. The most discriminant one was the probability of having all charged particles 
in the jet produced at the event interaction point. The use of this variable alone defined the impact-parameter technique. The additional variables were used only when a secondary vertex (SV) was reconstructed. These variables were, for all particles attached to the SV: the invariant mass, the fraction of the charged jet energy, the sum of all transverse momenta and the rapidity of each particle. The information from all five variables was combined into a single estimator $X_{\text {jet }}$ in an almost optimal way which provided discrimination between heavy and light jets with high purity and efficiency. To obtain $b$ (light)quark enriched samples, jets with an estimator value above (below) a given threshold $X_{\text {jet }} \geq X_{\text {jet }}^{b}\left(X_{\text {jet }}<X_{\text {jet }}^{\ell}\right)$ were selected. To tag events, the value of the two highest $b$ tagging jet variables were combined into an event estimator, $X_{\mathrm{ev}}=X_{\text {jet }}^{1}+X_{\text {jet }}^{2}$.

In the present analysis, this approach has been associated to a double-tag technique [28], which measures flavour-tagging efficiencies directly from data.

Using the two jets with highest $b$-tagging variables as the flavour jets (jets which are expected to contain a primary quark) makes no distinction between primary quarks originating in the $Z$ decay and secondary production of $b$ - and $c$-quarks from gluons $(g \rightarrow b \bar{b}, c \bar{c})$, a process referred to as gluon splitting and which constitutes a significant part of the systematic uncertainty in multi-jet flavourobservables (see Sect. 4.3).

To reduce the sample contamination from gluon splitting in four-jet events, the flavour jets were defined as follows: the most energetic jet in each event is identified as the first flavour jet. Remaining jets are ordered by angular proximity to it. The closest jet is discarded making the hypothesis that it is a gluon coming from the same primary quark. The second $b$ (light)-flavour jet is that with the highest $b$-tag (lowest $b$-tag) estimator among the two remaining jets. In this way, energy and angle information is combined to define the flavour-jets. As an additional selection, an event is not classified as $b \bar{b}$ if the most $b$-tagged jet is not among the two most energetic jets; this last selection reduces the uncertainty from $g_{b \bar{b}}$ and $g_{c \bar{c}}$ by a factor two. The effect of the remaining contamination due to gluon splitting is included in the gluon-splitting uncertainty and is well below the statistical uncertainties (Tables 4 and 5).

\subsection{Overview of the correction method}

\subsubsection{Event-tag}

To correct the two-jet observable $R_{2}^{b \ell}$ for detector effects and the flavour tagging procedure, the event-tag method described in reference $[2,3]$ was used:

$R_{2}^{b \ell}=\frac{\left[c_{B}^{\ell} d_{2 B}^{\ell}+R_{2}^{c \ell} c_{B}^{c} d_{2 B}^{c}\right]-\left[c_{L}^{\ell} d_{2 L}^{\ell}+R_{2}^{c \ell} c_{L}^{c} d_{2 L}^{c}\right] R_{2}^{b \ell-d e t}}{c_{L}^{b} d_{2 L}^{b} R_{2}^{b \ell-d e t}-c_{B}^{b} d_{2 B}^{b}}$,

where the measured rate $R_{2}^{b \ell-d e t}$ is corrected by using purities of the inclusive samples, $c_{Q}^{q}=N_{Q}^{q} / N_{Q}$ (the fraction of
Table 2. Flavour composition of the 1994 sample $\left(y_{\text {cut }}=\right.$ $0.0065)$. The number of events in the inclusive and 2-jet samples are shown separately for $B$ and $L=u d s$ tagged events for the chosen flavour-tagging working points. Purity and efficiency are also shown. Similar numbers were found with the 1995 data

\begin{tabular}{lccccc}
\hline & \multicolumn{3}{c}{ Event-tag method } & & \\
Flavour & Inclusive & 2 jets & cut & Purity & Efficiency \\
\hline$B$ & 111440 & 75147 & $X_{\mathrm{ev}} \geq 1.10$ & $98 \%$ & $38 \%$ \\
$L$ & 678282 & 414912 & $X_{\mathrm{ev}}<0.40$ & $73 \%$ & $58 \%$ \\
\hline
\end{tabular}

$q \bar{q}$ events tagged in the $Q$ category), and detector corrections taken from the DELSIM simulation, $d_{2 Q}^{q}=R_{2 Q}^{q} / R_{2}^{q}$ (where $R_{2 Q}^{q}$ is the two-jet rate of $q \bar{q}$ events tagged as $Q$ ). The factor $R_{2}^{c \ell}=R_{2}^{c} / R_{2}^{\ell}$ is taken from the simulation. Table 2 summarizes the number of events selected in the 1994 data in each flavour sample for the chosen working points of purity $P_{B}=c_{B}^{b}=98 \%\left(P_{L}=c_{L}^{\ell}=73 \%, L=\right.$ $u d s)$ and efficiency of $\epsilon_{B}^{b}=38 \%\left(\epsilon_{L}^{\ell}=58 \%\right)$ for $b$-flavoured (light-flavoured) events (where $\epsilon_{Q}^{q}=N_{Q}^{q} / N_{q}$, the ratio of tagged events of a given flavour to the total number of events of the same flavour), respectively.

The event-tag method has the advantage of applying the flavour-tagging procedure only in the inclusive sample, before events are classified into jet topologies.

\subsubsection{Double-jet tag}

The event-tag method, if the jet sample is topologically very different from the inclusive one, can introduce important biases. To prevent this, in the $R_{4}^{b \ell}$ measurement $b$-tagging is applied to jets. The observable in (2) is rewritten as:

$$
R_{4}^{b \ell}=\left(\frac{\Gamma(Z \rightarrow \ell \bar{\ell})}{\Gamma(Z \rightarrow b \bar{b})}\right) \frac{N_{4}^{b} / N_{4}}{N_{4}^{\ell} / N_{4}}=\left(\frac{1-R_{b}-R_{c}}{R_{b}}\right) \frac{N_{4}^{b}}{N_{4}^{\ell}} .
$$

The global normalisation can be obtained directly from the world average values of $R_{b}$ and $R_{c}$ [17]:

$$
\begin{aligned}
& R_{b}=0.21629 \pm 0.00066, \\
& R_{c}=0.1721 \pm 0.0030,
\end{aligned}
$$

which implies a $\pm 6 \%$ uncertainty on $R_{4}^{b \ell}$. A double-tag technique is used: the total number of four-jet events, $N_{4}$, the corresponding numbers for a given flavour $N_{4}^{q}, q=$ $b, u d s c$, and the tagging efficiencies $\epsilon_{B}^{b}$ and $\epsilon_{U D S C}^{u d s c}$ are obtained from comparing the number of four-jet events where two jets are tagged as $b$ or $u d s c$ to the number of events where a single jet is tagged. This is done by solving the following set of equations:

$$
\begin{aligned}
\mathcal{N}_{4} & =\left\{N_{4}^{b} \epsilon_{h}^{b}+\left(N_{4}-N_{4}^{b}\right) \epsilon_{h}^{\mathrm{non}-b}\right\}, \\
\frac{1}{2} \mathcal{N}_{4 B} & =\left\{N_{4}^{b} \epsilon_{h}^{b} \epsilon_{B}^{b}+\left(N_{4}-N_{4}^{b}\right) \epsilon_{h}^{\mathrm{non}-b} \epsilon_{B}^{\mathrm{non}-b}\right\}, \\
\mathcal{N}_{4 B B} & =\left\{N_{4}^{b} \epsilon_{h}^{b} \epsilon_{B B}^{b}+\left(N_{4}-N_{4}^{b}\right) \epsilon_{h}^{\mathrm{non}-b} \epsilon_{B B}^{\mathrm{non}-b}\right\},
\end{aligned}
$$


Table 3. Flavour composition of the 1994 sample $\left(y_{\text {cut }}=\right.$ $0.0065)$ tagged as $n$-jet $b$-quark $(B)$ and $u d s c$-quark events $(L)$ for the different jet topologies analysed, $n=2,3$ and 4 jets. Four-jet tagging uses the method described in Sect. 3.2 for the definition of flavour jets. Similar numbers were found with the 1995 data

\begin{tabular}{lrrccc}
\hline Topology & \multicolumn{5}{c}{ Double-tag method } \\
\hline jets $(B)$ & 136228 & \multicolumn{1}{c}{$Q Q$} & cut & Purity Efficiency \\
\hline jets $(L)$ & 640757 & 243716 & $X_{2 j} \geq+0.33$ & $92 \%$ & $57 \%$ \\
3 jets $(B)$ & 66034 & 15356 & $X_{3 j} \geq+0.19$ & $87 \%$ & $53 \%$ \\
3 jets $(L)$ & 362396 & 147605 & $X_{3 j}<-0.64$ & $93 \%$ & $84 \%$ \\
4 jets $(B)$ & 10720 & 2191 & $X_{4 j} \geq+0.05$ & $84 \%$ & $35 \%$ \\
4 jets $(L)$ & 91042 & 36773 & $X_{4 j}<-0.64$ & $89 \%$ & $86 \%$ \\
\hline
\end{tabular}

and equivalent equations for the $u d s c$-tagged samples. The left hand side of these equations are the measured quantities. $\mathcal{N}_{4}$ is the number of measured four-jet events. For each event the two jets which are most likely to contain a primary quark (flavour jets, see above) are selected and the flavour identification is done independently for both jets: $\mathcal{N}_{4 B}$ is the number of jets tagged as $B$ (with a maximum possible value of $2 \mathcal{N}_{4}$, two from each event) and $\mathcal{N}_{4 B B}$ is the number of events where the two flavour jets are simultaneously tagged as $B$. With this method, the jet-rates $R_{4}^{b}$ and $R_{4}^{\ell}$ are measured independently, together with the efficiencies $\epsilon_{B}^{b}$ and $\epsilon_{U D S C}^{u d s c}$. To accomplish this, double-jet tagging efficiencies $\epsilon_{Q Q}^{q}$ are related to the single jet-tagging efficiencies through correlation factors defined from

$$
\epsilon_{Q Q}^{q}=N_{Q Q}^{q} / N^{q} \equiv \epsilon_{Q}^{q} \epsilon_{Q}^{q}\left(1+\rho_{Q}^{q}\right) .
$$

Here, charm-events have been included in the udsc-tagged category: the light-quark content $N_{4}^{\ell}$ is extracted from $N_{4}^{u d s c}$ after dividing by a factor $\left(1+N_{4}^{c} / N_{4}^{\ell}\right)$ obtained from Monte Carlo event generators. Only hadronic eventselection efficiencies for each flavour, $\epsilon_{h}^{q}=N_{q}^{\text {sel }} / N_{q}$, mistagging efficiencies, $\epsilon_{Q}^{\text {non }-q}$ and $\epsilon_{Q Q}^{\text {non }-q}$, and flavour correlations for $b$ and light-tagging are computed from the simulation.

This procedure can be easily generalised to cover $n=2,3$-jet topologies in order to measure both jet-rates $\left(R_{n}^{b}, R_{n}^{\ell}\right)$ and the double-ratios $\left(R_{2,3}^{b \ell}\right)$ independently. Due to the $6 \%$ uncertainty from the global normalisation, the double-tag measurements for $R_{2,3}^{b \ell}$ are less precise than the corresponding event-tag result. However, they serve as a useful cross-check both of the final result and on the consistency between data and simulation for the flavourtagging efficiencies.

Results with this method have a better stability with respect to the value of the flavour-tagging threshold, and are more consistent with each other. ${ }^{3}$ The flavour composition of the 1994 sample is shown as an example in Tables 2

\footnotetext{
${ }^{3}$ From the relation $N_{4}=N_{4}^{b}+N_{4}^{u d s c}$, the double-ratio $R_{4}^{b \ell}$ can be obtained independently in two ways, starting either from $R_{4}^{b}$ or $R_{4}^{\ell}$.
}
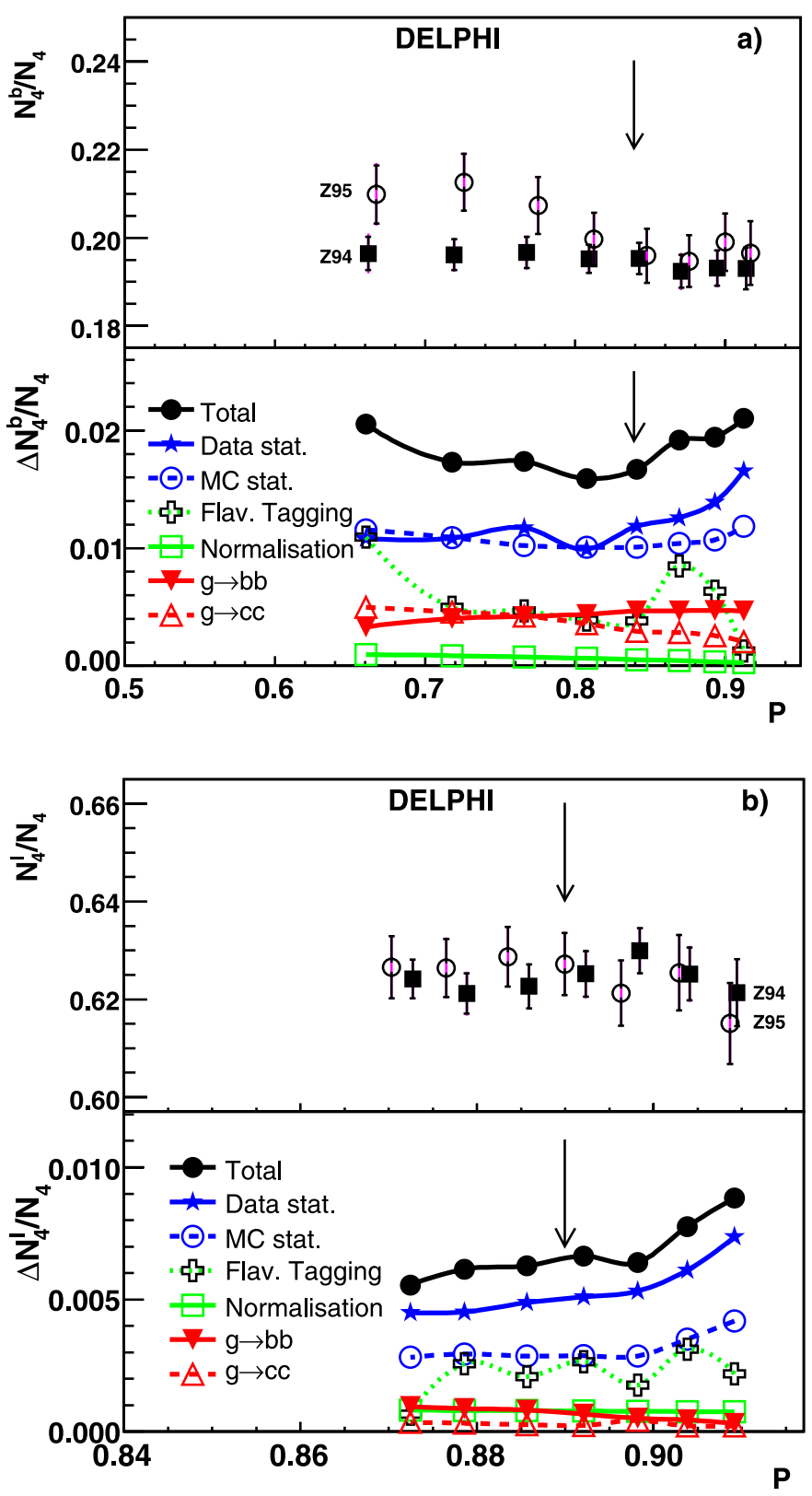

Fig. 1. Four-jet rate and its uncertainty as a function of a the $b$-purity and $\mathbf{b}$ the light-purity $\left(\ell=u d s c, y_{\text {cut }}=0.0065\right)$. Chosen working points are marked with arrows, and correspond to efficiencies of $\epsilon_{B}^{b}=35 \%$ and $\epsilon_{L}^{\ell}=86 \%$, respectively. The statistical (data and simulation) and total uncertainties are shown

and 3 . The stability obtained in the case of the four-jet rates is shown in Fig. 1 for the 1994 and 1995 data samples.

\section{Results}

The single-flavour jet rates $R_{n}^{q}, n=2,3,4$-jets, and the four-jet observable $R_{4}^{b \ell}$, are measured with the doubletag technique, while the two-jet observable $R_{2}^{b \ell}$ is measured using the event-tag method described in $[2,3]$. A de- 

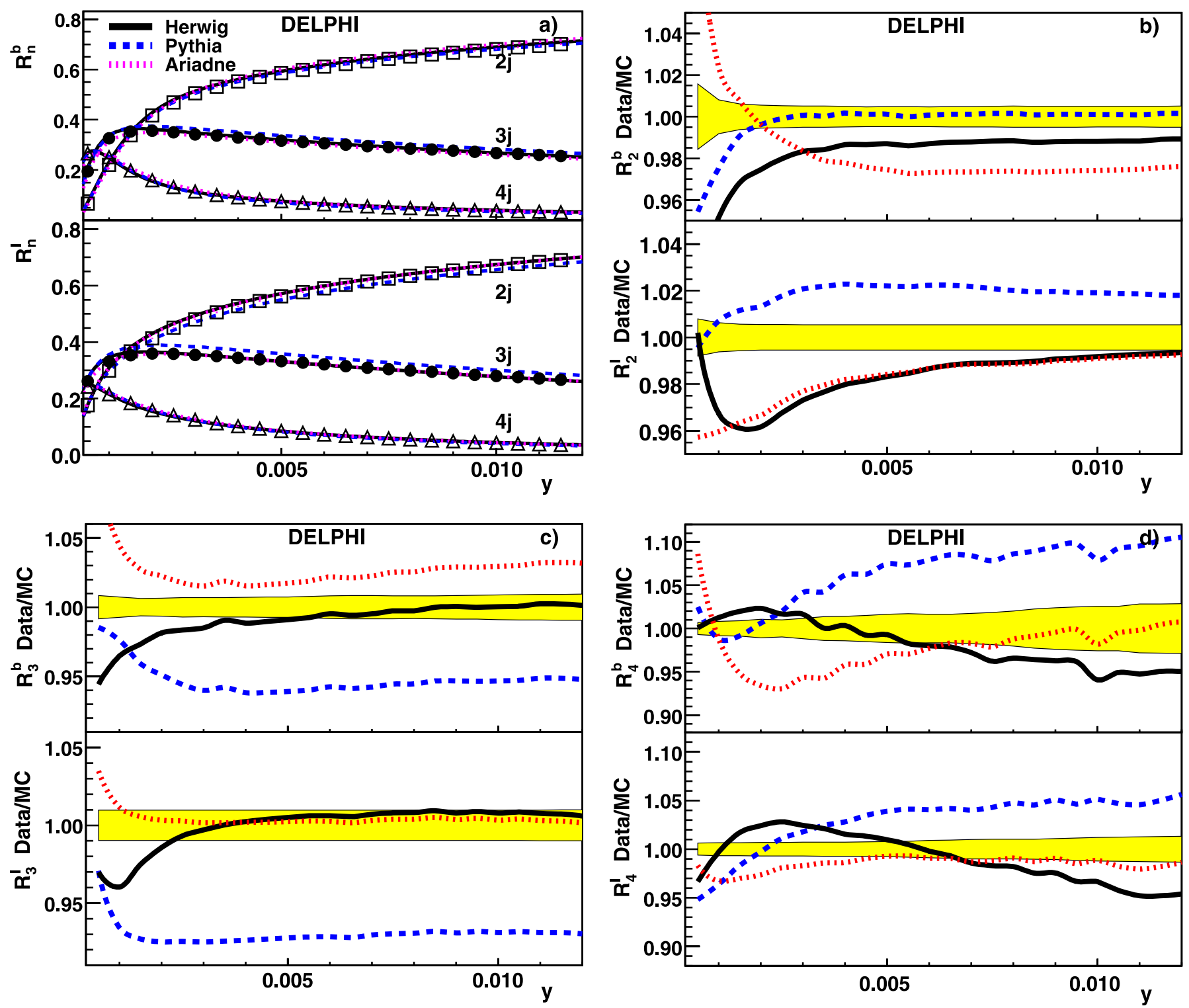

Fig. 2. Comparison between the measured $b$ and $\ell=u d s$ jet-rates and predictions from the PYTHIA 6.156, HERWIG 6.2 and ARIADNE 4.08 generators. b-d Ratio of data to the different generators. The shaded area shows the one standard deviation relative uncertainty (statistical and systematic added in quadrature) of the experimental measurement

scription of the experimental uncertainties considered in the analysis is given in Sect. 4.3. Theoretical uncertainties, arising in the comparison between ME predictions and the four-jet observable, are discussed in Sects. 4.5 and 4.6 .

\subsection{Single jet-rates, $R_{n}^{q}$}

The measured $R_{n}^{q}$ rates $(n=2,3,4$ jets, $q=b$ or $\ell=u d s)$ are shown in Fig. 2a together with predictions from the PYTHIA 6.156, HERWIG 6.2 and ARIADNE 4.08 generators tuned to DELPHI data $[24,25]$ (see Sect. 4.6 for the choice of the $b$-quark mass parameter in the generators). The detailed breakdown of the uncertainties of the measured jet-rates is shown in Table 4 . The $R_{3}^{\ell}$ measurements in 1994 and 1995 were found to be incompatible with each other at the two standard deviations level, indicating that some systematic effect was not taken into account in the three-jet light-quark rate. The systematic tagging uncertainty in $R_{3}^{\ell}$ was increased in order to fully cover this difference. Only the uncertainty in $R_{3}^{\ell}$ was increased since the $b$-tagging was developed from 2 -jet events yielding reliable $R_{2}^{\ell}$ results, and in 4 -jet events the $b$-tagging applies different cuts on angle and energy. The consistency of the experimental results and the prediction from the three event generators is shown in Fig. 2b-d. The HERWIG 6.2 and ARIADNE 4.08 generators provide a reasonable description of the six observables in the region of $y_{\text {cut }}$ between 0.001 and 0.010 . PYTHIA 6.156 gives the best description of $R_{2}^{b}$, but is inconsistent with the other jet-measurements at the three standard deviations level. 
Table 4. Breakdown of uncertainties for the $R_{n}^{q}$ jet-rate measurements at a reference $y_{\text {cut }}=0.0065$. The definition of each systematic contribution is given in Sect. 4.3

\begin{tabular}{lcccccc}
\hline & $R_{2}^{b}$ & $R_{2}^{\ell}$ & $R_{3}^{b}$ & $R_{3}^{\ell}$ & $R_{4}^{b}$ & $R_{4}^{\ell}$ \\
Value & 0.6224 & 0.6034 & 0.3004 & 0.3150 & 0.0598 & 0.0676 \\
\hline Statistical (data) & \pm 0.0019 & \pm 0.0008 & \pm 0.0016 & \pm 0.0006 & \pm 0.0007 & \pm 0.0004 \\
Statistical (sim.) & \pm 0.0012 & \pm 0.0005 & \pm 0.0009 & \pm 0.0004 & \pm 0.0006 & \pm 0.0003 \\
Tagging & \pm 0.0004 & \pm 0.0008 & \pm 0.0006 & \pm 0.0025 & \pm 0.0001 & \pm 0.0002 \\
Normalisation & \pm 0.0018 & \pm 0.0030 & \pm 0.0009 & \pm 0.0016 & \pm 0.0002 & \pm 0.0003 \\
$g_{b \bar{b}}$ & \pm 0.0003 & $<0.0001$ & \pm 0.0009 & $<0.0001$ & \pm 0.0003 & $<0.0001$ \\
$g_{c \bar{c}}$ & \pm 0.0006 & \pm 0.0002 & \pm 0.0010 & $<0.0001$ & \pm 0.0002 & $<0.0001$ \\
Total systematics & \pm 0.0020 & \pm 0.0031 & \pm 0.0017 & \pm 0.0030 & \pm 0.0004 & \pm 0.0004 \\
Total statistical & \pm 0.0023 & \pm 0.0009 & \pm 0.0018 & \pm 0.0007 & \pm 0.0009 & \pm 0.0005 \\
Total uncertainty & \pm 0.0030 & \pm 0.0033 & \pm 0.0025 & \pm 0.0031 & \pm 0.0010 & \pm 0.0006 \\
\hline
\end{tabular}

\subsection{Double-ratios, $R_{n}^{b \ell}$}

The measured double-ratios $R_{n}^{b \ell}(n=2,3,4$ jets) are shown in Figs. 3 and 4 together with predictions from the PYTHIA 6.156, HERWIG 6.2 and ARIADNE 4.08 generators tuned to DELPHI data $[24,25]$ (see Sect. 4.6 for the choice of the $b$-quark mass parameter in the generators).

Results for $R_{2}^{b \ell}$ and $R_{3}^{b \ell}$ from the event-tag and doubletag methods are shown in Fig. 3 (event-tag results for $R_{3}^{b \ell}$ are taken from [7]). $R_{2}^{b \ell}$ is not described well by either of the generators in the full $y_{\text {cut }}$ range. In all cases, both methods give consistent results within one standard deviation. A better experimental precision is found with the event-tag, because the global normalisation uncertainty is absent in this case and because flavourtagging uncertainties cancel to first order in the products $c_{Q}^{q} d_{n Q}^{q}$ (see (3)). Statistical uncertainties in the eventtag result are also smaller, as more data events are considered and as statistical fluctuations are partially re- duced in the ratios of the jet and inclusive samples. The detailed breakdown of the uncertainties of the measured double-ratios is shown in Table 5 for the event-tag method.

The $R_{4}^{b \ell}$ result with the double-tag method is shown in Fig. 4a, while the experimental systematics breakdown is summarized in Table 5. At $y_{\text {cut }}$ values above 0.004 the measurement is dominated by statistical uncertainties, while for very low values of $y_{\text {cut }}$ the data samples increase and the global normalisation uncertainty dominates. Gluon splitting uncertainties are kept low in the whole $y_{\text {cut }}$ range thanks to the dedicated anti-gluon splitting cut (see Sect. 3.2). HERWIG provides the best description, being compatible with the experimental data in the whole $y_{\text {cut }}$ range. However, the PYTHIA prediction is only 1.5 standard deviations away in the large $y_{\text {cut }}$ region; ARIADNE provides a good description of the data in the region $y_{\text {cut }} \geq 0.005$, while for lower values of $y_{\text {cut }}$ it tends to underestimate the mass effect.

Table 5. Breakdown of uncertainties for the $R_{n}^{b \ell}(n=2,3,4)$ double-ratio measurements. The three-jet result is taken from [7] and shown here for completeness. The two and three-jet measurements are based on the event-tag method, while $R_{4}^{b \ell}$ uses the double-tag technique as explained in Sect. 3.3. The $b$-mass values (running and pole) extracted from $R_{4}^{b \ell}$ at reference $y_{\text {cut }}=0.0065$ are also shown, both for the massive LO $\left(M_{b}=m_{b}\left(M_{Z}\right)\right)$ and approximate NLO calculations. Experimental and modelling uncertainties (experimental tuning and hadronisation model in the simulation) are detailed separately

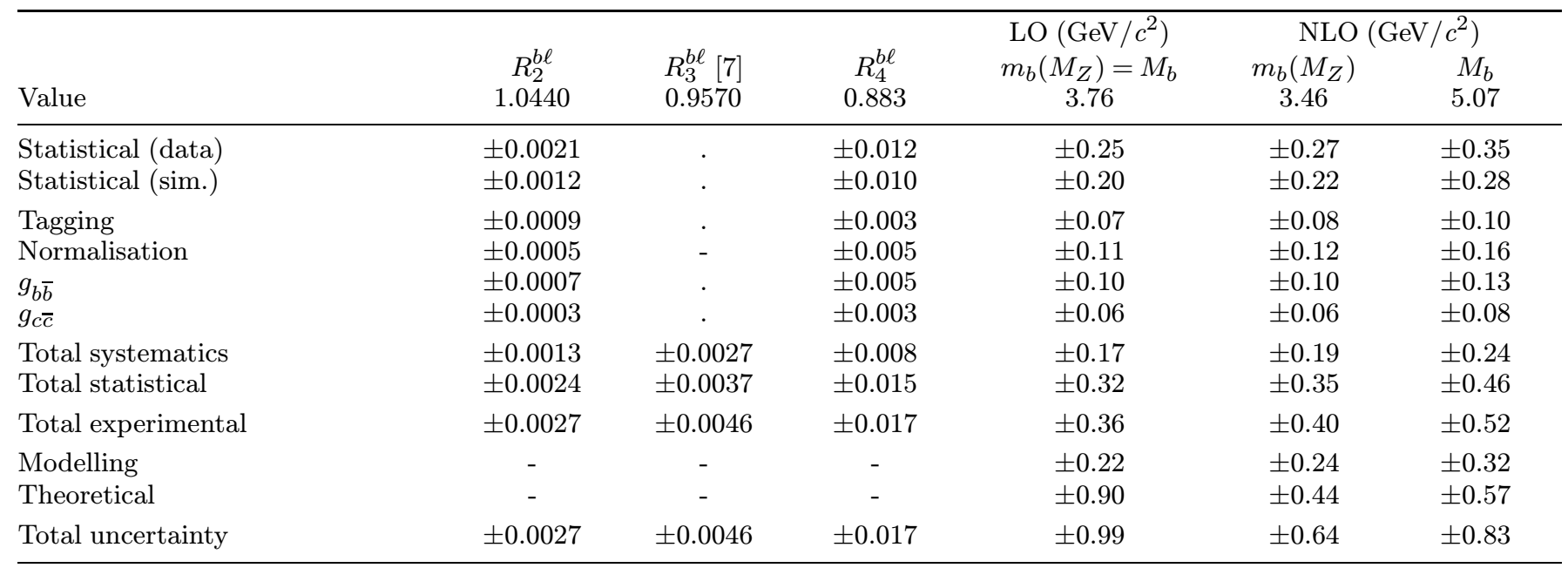



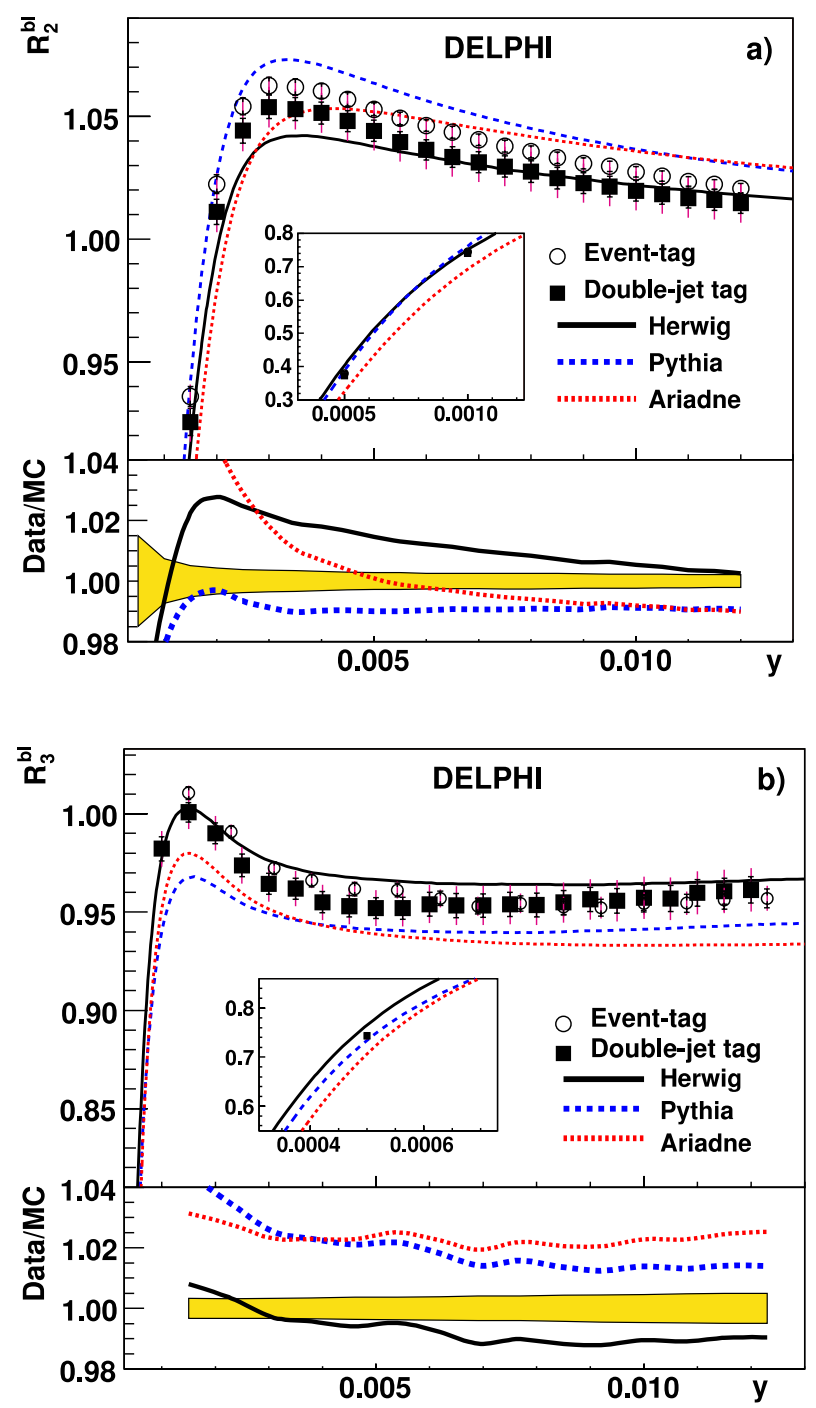

Fig. 3. Comparison between the event-tag (empty circles) and double-tag (full squares) techniques for the measured a $R_{2}^{b \ell}$ and b $R_{3}^{b \ell}$ observables. The event-tag result of $R_{3}^{b \ell}$ is taken from [7]. The combined statistical (inner bars) and total uncertainty of the experimental data are shown. The results are compared to the predictions from the HERWIG 6.2 (solid), PYTHIA 6.156 (dashed), and ARIADNE 4.08 (dotted) event generators. The lower insets of the plots show the ratio of data to the different generators. Also shown as the shaded area is the one standard deviation relative uncertainty (statistical and systematic added in quadrature) of the data

\subsection{Experimental uncertainties}

Experimental uncertainties arise in the process of correcting the detector-level measurement to hadron level, and are due to imperfections in the physics and detector modelling in the DELSIM simulation used in the correction procedure. The following sources have been considered in this analysis:

- Statistical: These uncertainties are due to the limited size of the experimental and simulated data samples. They are estimated from a toy simulation
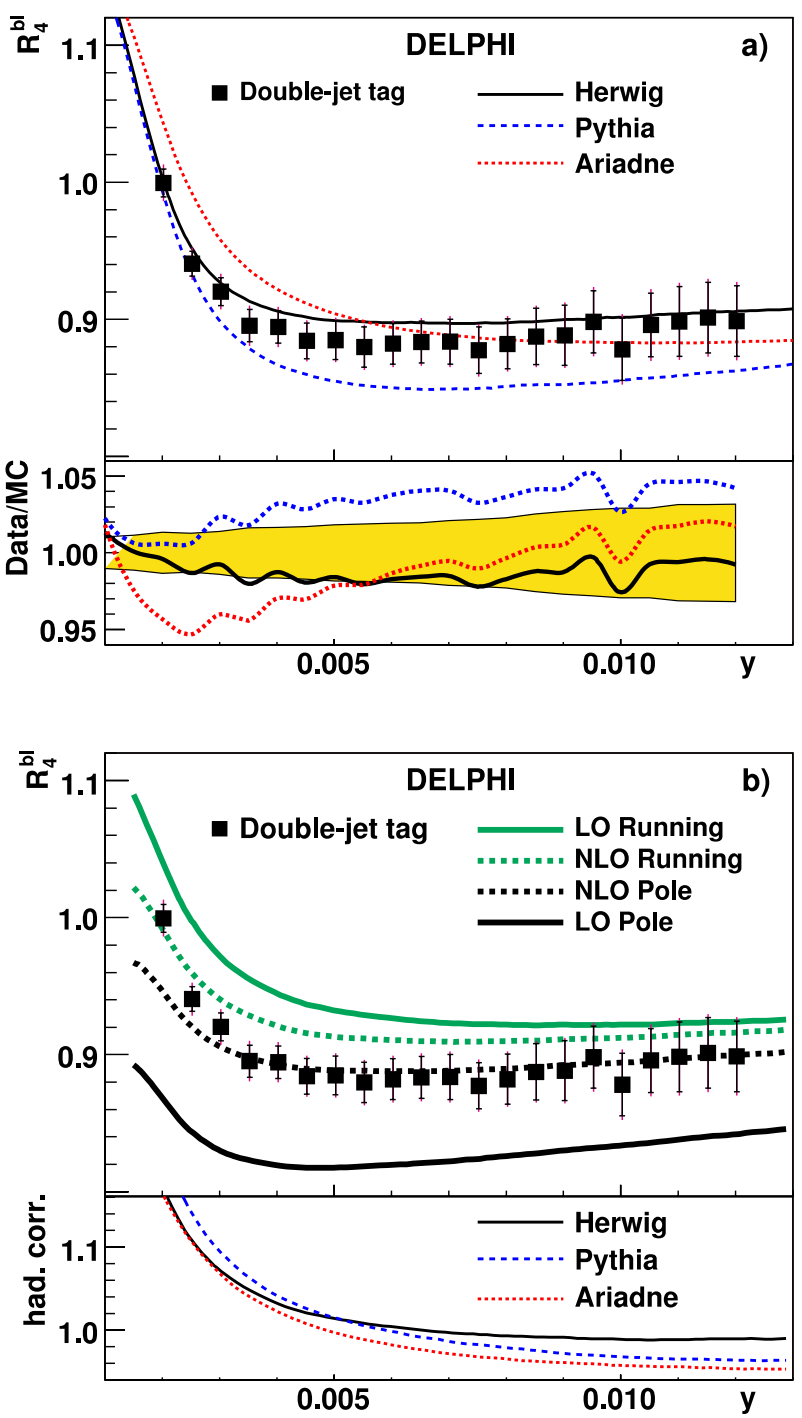

Fig. 4. a Comparison between the $R_{4}^{b \ell}$ measured with a double-tag technique and predictions from the HERWIG 6.2 (solid), PYTHIA 6.156 (dashed), and ARIADNE 4.08 (dotted) event generators. The combined statistical (inner bars) and total uncertainty of the experimental data are shown. The lower inset shows the relative deviation of the models to the data. Also shown as the shaded area is the total one standard deviation relative uncertainty (statistical and systematic added in quadrature) of the data. Below $y_{\text {cut }}=0.002$, the flavour tagging procedure fails and data results from the 1994 and 1995 data samples are not consistent with each other. b Comparison between the measured $R_{4}^{b \ell}$ and theoretical predictions: massive LO predictions and approximate (massless) NLO corrections for the pole and running $b$-quark mass definitions. Reference $b$-quark masses were obtained by evolving the average of low energy measurements $m_{b}\left(m_{b}\right)=4.20 \pm 0.07 \mathrm{GeV} / c^{2}[17]$ to the $M_{Z}$ scale as explained in Sect. 4.5. Hadronisation corrections, used to correct ME calculations, are shown for the three generators in the lower inset

based on Poisson statistics. Central values were taken from the data and simulated samples, and correlations between the different quantities were accounted 
for by building up the corresponding covariance matrix.

- Gluon splitting: The identification of primary $b$-quarks is based on the presence of long-lived $B$ and $D$-hadrons in the final state. However, light-quark events with gluon radiation splitting into secondary heavy quarks can produce a similar signature. The correction procedure is very sensitive to the gluon splitting rates in the Monte Carlo simulation through the signal and background efficiencies [7]. Their value was varied in the range of their quoted uncertainties [26] and the observed change in the observables was added in quadrature and taken to represent the corresponding uncertainty.

- Normalisation: The uncertainty on the global normalisation $R_{b} / R_{\ell}$ is estimated by varying the world average values of $R_{b}$ and $R_{\ell}=\left(1-R_{b}-R_{c}\right)$ in the range of their quoted uncertainties [17], and taking the maximum variation in the final observable as the global normalisation uncertainty. This results in a 6\%o relative uncertainty and is $y_{\text {cut }}$ independent. The uncertainty from the charm-/light-quark normalisation factor $\left(R_{n}^{c \ell}=R_{n}^{c} / R_{n}^{\ell}\right)$ is estimated as half the maximum difference obtained by using as input to the measurement the prediction from the three event generators used: PYTHIA 6.156, HERWIG 6.2 and ARIADNE 4.08 .

- Flavour-tagging: Signal efficiencies $\left(\epsilon_{n B}^{b}\right.$ and $\left.\epsilon_{n L}^{u d s c}\right)$ are measured from data and therefore do not contribute to the total uncertainty for the double-tag technique. To estimate the uncertainty due to the imperfect description of background efficiencies and flavour correlations $\left(\rho_{n Q}^{q}\right)$ in the simulation, the calibration of the $b$-tagging in the simulation was exchanged with the calibration obtained from data, which gives a poorer description of the lifetime probability [28]. Twice the observed difference was conservatively taken as the flavour-tagging uncertainty. For the event-tag technique, the related uncertainty was estimated as in [7] by varying the tagging efficiencies within their uncertainties: $\Delta \epsilon_{n B}^{b} / \epsilon_{n B}^{b}=$ $3 \%$ and $\Delta \epsilon_{n L}^{\ell} / \epsilon_{n L}^{\ell}=8 \%$ evaluated in reference [28]. The effect of mistagging efficiency was estimated by considering light-tagging as equivalent to anti $b$-tagging, i.e. $\Delta \epsilon_{n \ell}^{q}=\Delta \epsilon_{n b}^{q}$ for $q=b, c, \ell$ for the same cut value.

\subsection{Hadronisation corrections}

To compare parton-level fixed order ME calculations of $R_{4}^{b \ell-\text { part }}$ with experimental results, they must be corrected for hadronisation effects:

$$
R_{4}^{b \ell}=H_{4}^{b \ell} R_{4}^{b \ell-\text { part }} .
$$

The corrections $H_{4}^{b \ell}\left(y_{\text {cut }}\right)$ relating parton to hadron observables are taken to be linear bin-to-bin factors.

Three different generators, each tuned independently to the DELPHI data $[24,25]$, were used in this analysis: PYTHIA 6.156, HERWIG 6.2 and ARIADNE 4.08. It was found that the HERWIG and ARIADNE event generators are consistent both with the theoretical predictions at the parton level (within the theoretical uncertainty) and the data (see Fig. 4) for a large range of $y_{\text {cut }}$. The hadronisation corrections computed with the three generators are shown in Fig. $4 \mathrm{~b}$. The average of the HERWIG and ARIADNE predictions was used to correct the massive ME theoretical calculations (in the region of $y_{\text {cut }}$ studied here, the hadronisation correction computed from PYTHIA is contained in the band defined by the HERWIG and ARIADNE corrections).

\section{5 $b$-quark mass extraction and approximate NLO ME calculation}

For a given flavour $q$, the $n$-jet rate is defined as the normalised $n$-jet cross-section $R_{n}^{q}=\left[\Gamma_{n} / \Gamma_{\text {tot }}\right]^{Z \rightarrow q \bar{q}}$. Theoretically, it is convenient to use the double-ratios $R_{n}^{b \ell}=$ $R_{n}^{b} / R_{n}^{\ell}$ as in this observable most of the higher-order electroweak corrections, the first order dependence on $\alpha_{\mathrm{s}}$ and, to some extent also neglected higher-order terms in $\alpha_{\mathrm{s}}$, cancel out. Massive ME theoretical calculations exist up to order $\alpha_{\mathrm{s}}^{2}[14-16]$ and describe the 2, 3 and 4-jet rates for heavy $(b, c)$ and light quarks $(\ell=u d s)$. Such calculations, when performed in the on-shell scheme in terms of the pole mass, $M_{q}$, can be rewritten in terms of the running mass, $m_{q}$, defined in the $\overline{M S}$ scheme, using the following order $\alpha_{\mathrm{s}}$ relation:

$$
M_{q}^{2}=m_{q}^{2}(\mu)\left[1+\frac{\alpha_{\mathrm{s}}}{\pi}\left(\frac{8}{3}-2 \log \frac{m_{q}^{2}(\mu)}{\mu^{2}}\right)+\mathcal{O}\left(\alpha_{\mathrm{s}}^{2}\right)\right] .
$$

Both mass definitions are equivalent at LO (see (10)). For $y_{\text {cut }}=0.0065$, a value within a region with good stability, high sensitivity and small hadronisation corrections, the following $b$-quark mass value was obtained:

$$
\begin{aligned}
M_{b}= & m_{b}\left(M_{Z}\right)=3.76 \pm 0.32 \text { (stat) } \pm 0.17 \text { (syst) } \\
& \pm 0.22 \text { (had) } \pm 0.90 \text { (theo) } \mathrm{GeV} / c^{2} .
\end{aligned}
$$

The theoretical uncertainty is estimated as half the difference between the $R_{4}^{b \ell} \mathrm{LO}$ prediction for the running and pole $b$-quark mass definitions (see Fig. $4 \mathrm{~b}$ ).

To extract a meaningful $b$-quark running mass from the four-jet observable by means of (10), the NLO correction to $R_{n}^{q}$ would be needed, which is only available for massless quarks [18-20]. However, an improvement of the LO estimation can be obtained if most of the mass effect is contained in the LO term and hence the NLO correction to $R_{4}^{b \ell}$ can be approximated as massless [21]:

$$
R_{4}^{b \ell}=\frac{A^{b}\left(m_{b}\right) \alpha_{\mathrm{s}}^{2}+B^{\ell} \alpha_{\mathrm{s}}^{3}}{A^{\ell} \alpha_{\mathrm{s}}^{2}+B^{\ell} \alpha_{\mathrm{s}}^{3}},
$$

where the LO functions $A^{b}, A^{\ell}$ are taken from [14-16] and the NLO massless term $B^{\ell}$ from [18-20]. As for the case of $R_{3}^{b \ell}[29]$, it was found that:

- The NLO corrections using the pole and running mass definitions were both within the uncertainty band defined by the two LO curves. 

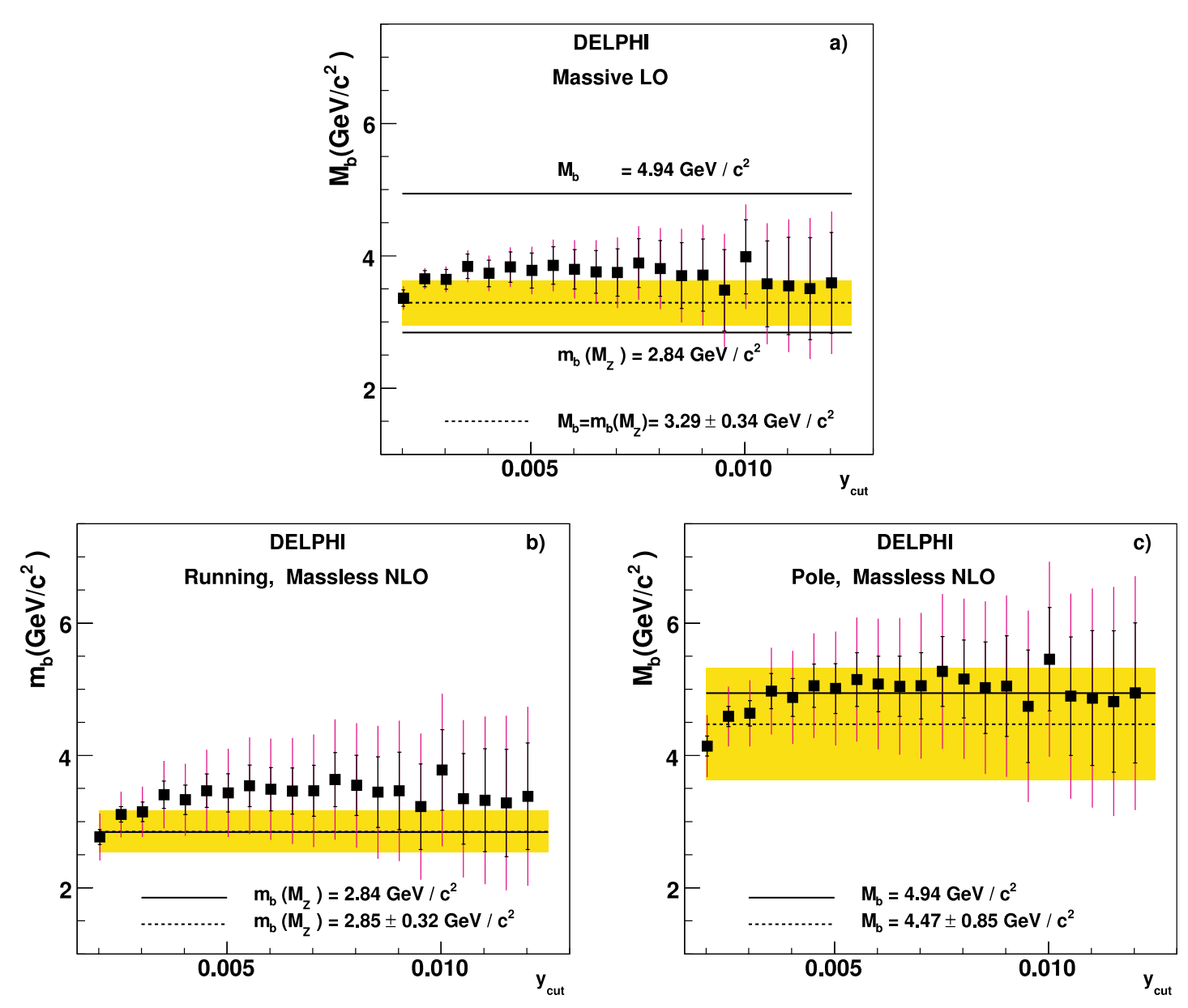

Fig. 5. a Massive LO results extracted from $R_{4}^{b \ell}$ (data points) compared with the result obtained at LO in the $R_{3}^{b \ell}$ analysis [7]: $M_{b}=m_{b}\left(M_{Z}\right)=3.29 \pm 0.34 \mathrm{GeV} / c^{2}$. In the LO result, no theoretical uncertainties are shown. Results obtained from $R_{4}^{b \ell}$ using massless NLO corrections include theoretical uncertainties estimated as explained in Sect. 4.5. They are shown for the $\mathbf{b}$ running and c pole mass definitions and are compared with the results obtained at NLO in the $R_{3}^{b \ell}$ analysis $[7]: m_{b}\left(M_{Z}\right)=2.85 \pm$ $0.32 \mathrm{GeV} / c^{2}$ and $M_{b}=4.47 \pm 0.85 \mathrm{GeV} / c^{2}$, respectively, shown as $\pm 1 \sigma$ shaded band with its central value as a dotted line. Predicted values from the QCD calculations at low energy, described in Sect. 4.5, are also shown as solid lines

- The running mass definition results in a smaller correction at NLO than the pole mass.

The $b$-mass values obtained from $R_{4}^{b \ell}$ using this approximation are shown in Fig. 5b and c. They are found to be stable in the region $y_{\text {cut }}>0.003$ and consistent with mass results obtained from $R_{3}^{b \ell}$ (both at LO and NLO) and predicted values from QCD calculations at low energy evolved to $M_{Z}$ using the RGE. For the running mass calculation, the massless NLO correction is small and results in very little effect. On the contrary, for the pole mass the NLO correction is about $10 \%$, leading to sizeable effects.

For the running $b$-quark mass definition, the theoretical prediction of $R_{4}^{b \ell}$ is taken to be the central value of the following, in principle equivalent, four calculations: (a) Full ratio as in (11), expressed in terms of the running mass by means of (10) at the scale $\mu=M_{Z}$; (b) Same, but using (10) at an arbitrary scale $\mu_{0}=M_{b}$ and evolving the result to $\mu=M_{Z}$ via the RGE to obtain $m_{b}\left(M_{Z}\right)$; (c) Series expansion of (11), expressed in terms of $m_{b}\left(M_{Z}\right)$ as in the first method; (d) Same, but introducing an arbitrary intermediate scale as in the second method. The pole mass prediction is obtained in a similar way. The resulting predictions for $R_{4}^{b \ell}$ are shown in Fig. 4b for a reference $b$-quark mass obtained by evolving the average of low energy measurements $m_{b}\left(m_{b}\right)=4.20 \pm 0.07 \mathrm{GeV} / c^{2}$ [17] to the $M_{Z}$ scale, $m_{b}\left(M_{Z}\right)=2.84 \pm 0.06 \mathrm{GeV} / c^{2}$, or by translating it to a pole mass value: $M_{b}=4.94 \pm 0.08 \mathrm{GeV} / c^{2}$. The strong coupling constant value used was $\alpha_{\mathrm{s}}\left(M_{Z}\right)=$ $0.1202 \pm 0.0050[30]$.

\subsection{Theoretical and modelling uncertainties}

The following sources of systematic uncertainty have been considered for the comparison of the corrected four-jet ME calculations with the experimental results:

- Theoretical uncertainties, due to missing higher orders in matrix element calculations and to the use of massless next-to-leading corrections for the mass extraction, 
Table 6. Summary of experimental two and three-jet rates, with their total uncertainty, as a function of $y_{\text {cut }}[8]$

\begin{tabular}{lccccc}
\hline$y_{\text {cut }}$ & $R_{2}^{b}$ & $R_{2}^{\ell}$ & $R_{2}^{b \ell}$ & $R_{3}^{b}$ & $R_{3}^{\ell}$ \\
\hline 0.003 & $0.505 \pm 0.003$ & $0.481 \pm 0.003$ & $1.062 \pm 0.004$ & $0.342 \pm 0.002$ & $0.355 \pm 0.003$ \\
0.004 & $0.553 \pm 0.003$ & $0.527 \pm 0.003$ & $1.060 \pm 0.003$ & $0.329 \pm 0.002$ & $0.345 \pm 0.003$ \\
0.005 & $0.585 \pm 0.003$ & $0.562 \pm 0.003$ & $1.053 \pm 0.003$ & $0.317 \pm 0.002$ & $0.333 \pm 0.003$ \\
0.006 & $0.611 \pm 0.003$ & $0.591 \pm 0.003$ & $1.046 \pm 0.003$ & $0.306 \pm 0.003$ & $0.321 \pm 0.003$ \\
0.007 & $0.633 \pm 0.003$ & $0.615 \pm 0.003$ & $1.040 \pm 0.003$ & $0.295 \pm 0.003$ & $0.310 \pm 0.003$ \\
0.008 & $0.651 \pm 0.003$ & $0.635 \pm 0.003$ & $1.040 \pm 0.003$ & $0.286 \pm 0.002$ & $0.300 \pm 0.003$ \\
0.009 & $0.667 \pm 0.003$ & $0.653 \pm 0.003$ & $1.031 \pm 0.003$ & $0.277 \pm 0.002$ & $0.289 \pm 0.003$ \\
0.010 & $0.681 \pm 0.003$ & $0.669 \pm 0.004$ & $1.027 \pm 0.002$ & $0.268 \pm 0.002$ & $0.280 \pm 0.003$ \\
\hline
\end{tabular}

cannot be rigorously estimated in the case of four-jets. However, following a comparison between the same approximation applied to $R_{3}^{b \ell}$ with the full massive calculation available in this case, this uncertainty was conservatively taken to be twice the maximum difference between the four predictions defined in Sect. 4.5. The theoretical uncertainty is responsible for about $0.4-0.5 \mathrm{GeV} / c^{2}$ in the uncertainty of the final result, and it is almost independent of $y_{\text {cut }}$. Although lower than in the case of the LO calculation, it is three times higher than in the completely massive three-jet calculation.

- Modelling uncertainties, related to the correction for hadronisation effects of the theoretical calculations at parton level using Monte Carlo event generators. This includes the uncertainty on the tuned values of the free parameters in each model (including the $b$-mass parameter entering in the parton shower [7]) and the modelling of hadronisation. The size of the modelling uncertainty is estimated as half the difference between the predictions from HERWIG 6.2 and ARIADNE 4.08. To include the $b$-mass uncertainty in the estimation of the hadronisation systematic uncertainty, the mass parameter in HERWIG and ARIADNE was varied within $\pm 0.125 \mathrm{GeV} / c^{2}$ around their central values in order to maximize the difference between both predictions. This was achieved by setting the mass parameter to $M_{b}=4.85 \mathrm{GeV} / c^{2}$ in both generators. The total modelling uncertainty amounts to $\pm(1-2) \%$ in the region of $y_{\text {cut }}>0.004$, corresponding to about $\pm 0.2 \mathrm{GeV} / c^{2}$ in terms of both the running and pole mass results. The contribution from varying the mass parameter amounts to about $\pm 0.1 \mathrm{GeV} / c^{2}$.

The breakdown of the theoretical and modelling uncertainties in the $b$-quark mass results obtained from $R_{4}^{b \ell}$ is detailed in Table 5 .

\section{Summary and conclusions}

A new determination of the hadron-level $R_{n}^{b}$ and $R_{n}^{\ell=u d s}$ jet-rates ( $n=2,3,4$ jets) has been performed, using flavour

\footnotetext{
4 The result obtained with the PYTHIA 6.156 event-generator is compatible with the quoted results within the modelling uncertainty.
}

tagging only in each $n$-jet sample and obtaining the global normalisation of the observables from the world average $R_{b}$ and $R_{c}$ measurements [17]. This measurement is based on a double-tag technique which measures the flavourtagging efficiencies directly from data, thereby reducing systematic uncertainties.

Double-ratio observables are also studied: $R_{4}^{b \ell}$ is obtained from the four-jet rates $R_{4}^{b}$ and $R_{4}^{\ell}$ using this doubletag technique, and $R_{2}^{b \ell}$ using the event-tag method defined in reference [7]. Results from $R_{2}^{b \ell}$ (and from the previous measurements of $R_{3}^{b \ell}$ in [7]) are also cross-checked.

Results are presented at hadron level, in order to allow for future comparisons without having to unfold hadronisation and detector corrections applied to the data (a summary of jet-rate results as a function of $y_{\text {cut }}$ is shown in Tables 6 and 7). They are compared to three Monte Carlo event generators: PYTHIA 6.156, HERWIG 6.2 and ARIADNE 4.08, tuned to DELPHI data $[24,25]$. The HERWIG 6.2 generator gives the best overall description of flavour jet-rates, $R_{n}^{b}$ and $R_{n}^{\ell}$, but ARIADNE 4.08 provides the best results for $R_{n}^{\ell}$. For double-ratios, HERWIG 6.2 gives also the best description. However, the two-jet observable $R_{2}^{b \ell}$ is not satisfactorily described by any of the three generators considered.

A new determination of the $b$-quark mass in the four-jet topology has been performed using the CAMBRIDGE jetclustering algorithm [8]. The mass is measured by comparing the experimental results of $R_{4}^{b \ell}$ at $y_{\text {cut }}=0.0065$ with fixed order ME massive LO calculations assuming the universality of the strong coupling constant, $\alpha_{\mathrm{s}}$. The measured

Table 7. Summary of experimental four-jet rates, with their total uncertainty, as a function of $y_{\text {cut }}[8]$

\begin{tabular}{lccc}
\hline$y_{\text {cut }}$ & $R_{4}^{b}$ & $R_{4}^{\ell}$ & $R_{4}^{b \ell}$ \\
\hline 0.003 & $0.1148 \pm 0.0013$ & $0.1248 \pm 0.0009$ & $0.920 \pm 0.013$ \\
0.004 & $0.0911 \pm 0.0012$ & $0.1018 \pm 0.0007$ & $0.895 \pm 0.015$ \\
0.005 & $0.0757 \pm 0.0012$ & $0.0856 \pm 0.0007$ & $0.885 \pm 0.016$ \\
0.006 & $0.0642 \pm 0.0011$ & $0.0729 \pm 0.0007$ & $0.882 \pm 0.017$ \\
0.007 & $0.0555 \pm 0.0010$ & $0.0628 \pm 0.0006$ & $0.884 \pm 0.019$ \\
0.008 & $0.0486 \pm 0.0010$ & $0.0551 \pm 0.0006$ & $0.88 \pm 0.03$ \\
0.009 & $0.0432 \pm 0.0010$ & $0.0486 \pm 0.0005$ & $0.89 \pm 0.02$ \\
0.010 & $0.0380 \pm 0.0010$ & $0.0431 \pm 0.0005$ & $0.88 \pm 0.03$ \\
\hline
\end{tabular}


value is:

$$
\begin{aligned}
m_{b}\left(M_{Z}\right)= & 3.76 \pm 0.32(\text { stat }) \pm 0.17 \text { (syst) } \pm 0.22(\text { had }) \\
& \pm 0.90(\text { theo }) \mathrm{GeV} / c^{2} .
\end{aligned}
$$

A procedure to approximate the NLO corrections with the massless component in order to improve the result has been tested successfully with the three-jet massive calculations. The measured value of the running $b$-quark mass when applying this method to the four-jet observable is:

$$
\begin{aligned}
m_{b}\left(M_{Z}\right)= & 3.46 \pm 0.35(\text { stat }) \pm 0.19(\text { syst }) \pm 0.24(\bmod ) \\
& \pm 0.44(\text { theo }) \mathrm{GeV} / c^{2}
\end{aligned}
$$

and the corresponding value for the pole mass is:

$$
\begin{aligned}
M_{b}= & 5.07 \pm 0.46(\text { stat }) \pm 0.24(\text { syst }) \pm 0.32(\bmod ) \\
& \pm 0.57(\text { theo }) \mathrm{GeV} / c^{2} .
\end{aligned}
$$

These results agree within the uncertainties with the values obtained evolving the average of low energy measurements $m_{b}\left(m_{b}\right)=4.20 \pm 0.07 \mathrm{GeV} / c^{2}[17]$ to the $M_{Z}$ scale using the RGE: $m_{b}\left(M_{Z}\right)=2.84 \pm 0.06 \mathrm{GeV} / c^{2}$, or by translating it to a pole mass value: $M_{b}=4.94 \pm 0.08 \mathrm{GeV} / c^{2}$. The values of $m_{b}\left(M_{Z}\right)$ obtained from the $\mathrm{LO}$ and approximate NLO $R_{4}^{b \ell}$ calculations are shown in Fig. 6 together with results from other measurements at the $M_{Z}$ scale, in particular the most precise result from $R_{3}^{b \ell}, m_{b}\left(M_{Z}\right)=2.85 \pm$

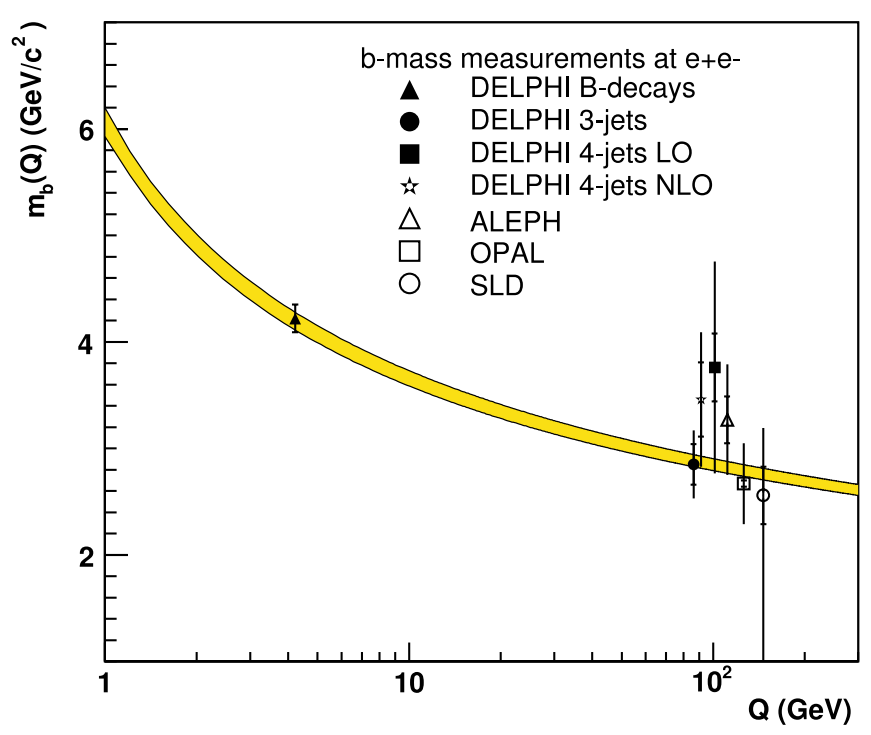

Fig. 6. The energy evolution of the $\overline{M S}$-running $b$-quark mass $m_{b}(Q)$ as measured at LEP. DELPHI results from $R_{3}^{b \ell}[7]$ at the $M_{Z}$ scale and from semileptonic $B$-decays [31] at low energy are shown together with results from other experiments (ALEPH [4], OPAL [5] and SLD [6]). The masses extracted from LO and approximate NLO calculations of $R_{4}^{b \ell}$ are found to be consistent with previous experimental results and with the reference value $m_{b}(Q)$ (grey band) obtained from evolving the average $m_{b}\left(m_{b}\right)=4.20 \pm 0.07 \mathrm{GeV} / c^{2}$ from [17] using QCD RGE (with a strong coupling constant value $\alpha_{\mathrm{S}}\left(M_{Z}\right)=$ $0.1202 \pm 0.0050[30])$
$0.32 \mathrm{GeV} / c^{2}$ [7], as well as results at low energy from semileptonic $B$-decays [31] obtained at a lower mass scale. All experimental results are consistent with each other assuming the QCD running prediction from RGE.

The main limitation in the extraction of $m_{b}\left(M_{Z}\right)$ from the $R_{4}^{b \ell}$ measurement is theoretical. If a calculation with resummed $L L$ logarithms [32,33] could be used, a larger range of $y_{\text {cut }}$ could be exploited. This could potentially lead to a lower uncertainty.

Improvements to the precision of $m_{b}\left(M_{Z}\right)$ are not expected from combining the different measurements because they are largely limited by common systematic uncertainties. Other methods will likely be needed at future colliders in order to obtain more precise determinations of the $b$-quark mass at high energy. This will be important to interpret the precise measurements at the Linear Collider in searches for new physics. As an example, a future linear collider operating at $\sqrt{s}=500 \mathrm{GeV}$ will produce Higgs bosons copiously (if they exist). Since the decay branching fraction into $b$-quarks is expected to be proportional to the mass squared, measurements of this decay channel would be very sensitive to the exact value of the mass at that scale.

Acknowledgements. We are grateful to G. Rodrigo and A. Santamaría for providing the theoretical input for this measurement. We are also indebted to T. Sjöstrand for his help in understanding how mass effects are implemented in PYTHIA. We would also like to thank G. Dissertori for continuous advice and J. Portoles and M. Eidemüller for their information about the $b$ pole mass.

We are greatly indebted to our technical collaborators, to the members of the CERN-SL Division for the excellent performance of the LEP collider, and to the funding agencies for their support in building and operating the DELPHI detector. We acknowledge in particular the support of Austrian Federal Ministry of Education, Science and Culture, GZ 616.364/2-III/2a/98, FNRS-FWO, Flanders Institute to encourage scientific and technological research in the industry (IWT) and Belgian Federal Office for Scientific, Technical and Cultural affairs (OSTC), Belgium, FINEP, CNPq, CAPES, FUJB and FAPERJ, Brazil, Ministry of Education of the Czech Republic, project LC527, Academy of Sciences of the Czech Republic, project AV0Z10100502, Commission of the European Communities (DG XII), Direction des Sciences de la Matière, CEA, France, Bundesministerium für Bildung, Wissenschaft, Forschung und Technologie, Germany, General Secretariat for Research and Technology, Greece, National Science Foundation (NWO) and Foundation for Research on Matter (FOM), The Netherlands, Norwegian Research Council, State Committee for Scientific Research, Poland, SPUB-M/CERN/PO3/DZ296/2000, SPUBM/CERN/PO3/DZ297/2000, 2P03B 10419 and 2P03B 69 23(2002-2004), FCT - Fundação para a Ciência e Tecnologia, Portugal, Vedecka grantova agentura MS SR, Slovakia, Nr. 95/5195/134, Ministry of Science and Technology of the Republic of Slovenia, CICYT, Spain, AEN99-0950, AEN99-0761 and IN2P3/CYCIT bilateral funding agreement PP01/1, The Swedish Research Council, Particle Physics and Astronomy Research Council, UK, Department of Energy, USA, DE-FG02- 
01ER41155, EEC RTN contracts HPRN-CT-00292-2002 and RTN2-2001-00450.

\section{References}

1. G. Rodrigo, M. Bilenky, A. Santamaría, Nucl. Phys. B 439, 505 (1995)

2. DELPHI Collaboration, P. Abreu et al., Phys. Lett. B 418, 430 (1998)

3. S. Martí i García, J. Fuster, S. Cabrera, Nucl. Phys. B Proc. Suppl. 64, 376 (1998)

4. ALEPH Collaboration, R. Barate et al., Eur. Phys. J. C 18, 1 (2000)

5. OPAL Collaboration, G. Abbiendi et al., Eur. Phys. J. C 21, 411 (2001)

6. A. Brandenburg et al., Phys. Lett. B 468, 168 (1999)

7. DELPHI Collaboration, J. Abdallah et al., Eur. Phys. J. C 46, 569 (2006)

8. Y.L. Dokshitzer et al., JHEP 9708, 001 (1997)

9. T. Sjöstrand et al., Comput. Phys. Commun. 135, 238 (2001)

10. T. Sjöstrand et al., PYTHIA 6.2 Physics and Manual, hep-ph/0108264

11. G. Marchesini et al., Comput. Phys. Commun. 67, 465 (1992)

12. G. Corcella et al., JHEP 0101, 010 (2001)

13. L. Lönnblad, Comput. Phys. Commun. 71, 15 (1992)

14. G. Rodrigo, M. Bilenky, A. Santamaría, Phys. Rev. Lett. 79, 193 (1997)
15. W. Bernreuther, A. Brandenburg, P. Uwer, Phys. Rev. Lett. 79, 189 (1997)

16. P. Nason, C. Oleari, Phys. Lett. B 407, 57 (1997)

17. Particle Data Group, W.-M. Yao et al., J. Phys. G 33, 1 (2006)

18. Z. Nagy, Z. Trocsanyi, Phys. Rev. Lett. 79, 3604 (1997)

19. Z. Nagy, Z. Trocsanyi, Phys. Rev. D 59, 014020 (1999)

20. Z. Nagy, Z. Trocsanyi, Phys. Rev. D 62, 099902 (2000) [Erratum]

21. J. Drees, G. Rodrigo, private communication

22. DELPHI Collaboration, P. Aarnio et al., Nucl. Instrum. Methods A 303, 233 (1991)

23. DELPHI Collaboration, P. Abreu et al., Nucl. Instrum. Methods A 378, 57 (1996)

24. DELPHI Collaboration, P. Abreu et al., Z. Phys. C 73, 11 (1996)

25. for the tuning of HERWIG 6.2, see [7]

26. The LEP/SLD Heavy Flavour Working group, LEPHF/ 2001-01, http://lepewwg.web.cern.ch/LEPEWWG/heavy/ lephf0101.ps.gz

27. DELPHI Collaboration, J. Abdallah et al., Eur. Phys. J. C 32, 185 (2004)

28. DELPHI Collaboration, P. Abreu et al., Eur. Phys. J. C 10, 415 (1999)

29. M. Bilenky et al., Phys. Rev. D 60, 114006 (1999)

30. The LEP QCD Working Group, paper in preparation

31. M. Battaglia et al., Phys. Lett. B 556, 41 (2003)

32. F. Krauss, G. Rodrigo, Phys. Lett. B 576, 135 (2003)

33. S. Catani et al., Nucl. Phys. B 627, 189 (2002) 